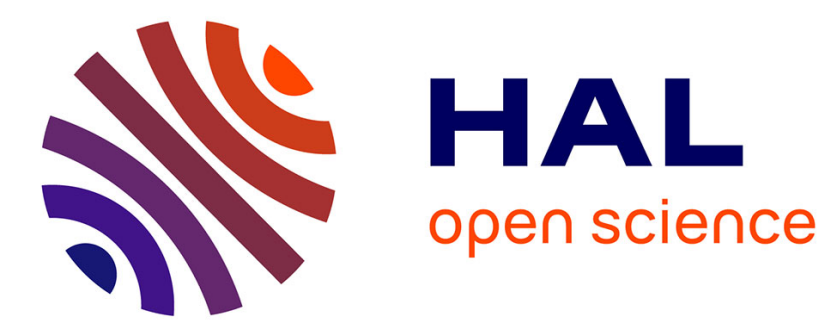

\title{
Convergence of a multidimensional Glimm-like scheme for the transport of fronts
}

Thierry Gallouët, Olivier Hurisse

\section{To cite this version:}

Thierry Gallouët, Olivier Hurisse. Convergence of a multidimensional Glimm-like scheme for the transport of fronts. IMA Journal of Numerical Analysis, 2021. hal-02940407v2

\section{HAL Id: hal-02940407 \\ https://hal.science/hal-02940407v2}

Submitted on 26 Jan 2021

HAL is a multi-disciplinary open access archive for the deposit and dissemination of scientific research documents, whether they are published or not. The documents may come from teaching and research institutions in France or abroad, or from public or private research centers.
L'archive ouverte pluridisciplinaire HAL, est destinée au dépôt et à la diffusion de documents scientifiques de niveau recherche, publiés ou non, émanant des établissements d'enseignement et de recherche français ou étrangers, des laboratoires publics ou privés. 


\title{
Convergence of a multidimensional Glimm-like scheme for the transport of fronts.
}

\author{
THIERRY GallouËt * \\ Institut de Mathématiques de Marseille, Aix-Marseille Université, \\ Technopôle Château-Gombert, 39 rue Frédéric Joliot-Curie, 13453 Marseille, France \\ AND \\ OLIVIER Hurisse ${ }^{\dagger}$ \\ EDF Lab Chatou, 6 quai Watier, 78400 Chatou, France
}

\begin{abstract}
This paper is devoted to the numerical analysis of a numerical scheme dedicated to the simulation of front advection. The latter has been recently proposed and it is based on the ideas used for the Glimm's scheme. It relies on a two-step approach: a convection step is followed by a projection step which is based on a random choice. The main advantage of this scheme is that it applies to multi-dimensional problems. In the present paper a convergence result for this scheme is provided for a particular class of multi-dimensional problems. Glimm's scheme, front propagation, multidimensional problem, random choice.
\end{abstract}

\section{Introduction}

In [8], a scheme based on the idea of $[5,1,2,3,6]$ has been introduced in order to deal with the advection of indicator functions. The whole algorithm is very simple and it relies on a two-step approach for which a convection step is followed by a projection step. For the convection step, the approximated solution is updated using a classical scheme. In the present work, the Upwind scheme has been chosen but it should be noted that the convection step may be performed with other schemes, as shown in [8]. The front of the approximated function is smeared by the numerical diffusion associated with the convection scheme. Therefore, a second step is introduced for stiffening this smeared profile. Its aim is indeed to recover an approximated solution which corresponds to the approximation of an indicator function (i.e. that for each cell of the mesh can only take two values). This second step, or Glimm Random Update (GRU), is performed on the basis of a random choice.

The whole scheme proposed in [8], and studied in details here, relies on the analysis of the Upwind scheme that has been carried out in [4] or in [10]. In these references, a probabilistic analysis of the classical finitevolume Upwind scheme is performed. The approximated solution of the latter can be built as the expectation of a stochastic process, where the characteristics are stochastic and follow a Markov chain. It arises from this analysis that the effective rate of convergence of $1 / 2$ of the Upwind scheme can be associated to the fluctuations of the stochastic characteristics around the average characteristic, and to the expectation operator applied to recover the Upwind scheme. Following Glimm's idea, only one sample of this stochastic process is selected by the GRU step, which avoids to apply for an expectation operator. Doing so, we could also expect to get an effective convergence rate higher than 1/2 and we might expect a better accuracy for the approximated solutions. The GRU step can thus be seen as the random choice of one sample of an underlying stochastic process for the characteristics, whose transition probabilities are obtained by the scheme used for the convection step. This scheme has been tested in [8] on several two-dimensional test cases, considering structured and unstructured meshes. Actually, for all these test cases, an effective rate of convergence around 0.8 has been observed for the Upwind-GRU scheme, whereas a classical first-order scheme has an effective rate of convergence of $1 / 2$ on such test cases. Beyond this, it has also been shown that the GRU step improves the accuracy of the approximated solutions on coarse meshes. It seems that it allows to get for two-dimensional cases the same efficiency than the classical Glimm's scheme in the one-dimensional setting.

Unlike for the Glimm's scheme, the GRU step is dedicated to a limited class of problems. But the main advantage of the GRU step is that it can be applied to multi-dimensional advection problems, as shown in [8]. Although the method can be applied in a three-dimensional framework, we focus here on the advection of a scalar quantity $\Phi$ in a two-dimensional space whose coordinates are $x$ and $y$. We assume that $\Phi$ can only take

\footnotetext{
*Email: thierry.gallouet@univ-amu.fr

$\dagger$ Corresponding author. Email: olivier.hurisse@edf.fr
} 
two different values at time $t$ and point $(x, y)$, say 0 and 1 , so that it represents an indicator function of a sub-domain of $\mathbb{R}^{2}$. Even if the GRU step can handle more complex advection problems, we restrict our study here to the simple case with a constant and uniform advection velocity field $\left(U_{x}, U_{y}\right)$. Hence, the system of equations we are concerned with can be written:

$$
\left\{\begin{array}{l}
\partial_{t} \Phi(t, x, y)+U_{x} \partial_{x} \Phi(t, x, y)+U_{y} \partial_{y} \Phi(t, x, y)=0 \\
\Phi(t=0, x, y)=\Phi^{0}(x, y)
\end{array}\right.
$$

where the initial condition $\Phi^{0}(x, y)$ is an indicator function for which the domain with value 1 and the domain with the value 0 are separated by a planar front. The scheme examined in this paper is based on the GRU step and on the Upwind scheme for the convection step. It is denoted herein by Upwind-GRU.

As mentioned in [8], for the one-dimensional counterpart of the advection problem described above, the Upwind-GRU scheme and the Glimm's scheme give the same update formulae. Hence, in this case, the proof of convergence for the Glimm's scheme is sufficient to prove the convergence of the Upwind-GRU scheme. Therefore, we focus in this work on the two-dimensional case which is more specific to the GRU step. Indeed, for the particular two-dimensional problem described above, some theoretical results can be exhibited. In particular a proof that the approximated solutions statistically converge towards the exact solution is proposed.

In [5] - when considering the Glimm's scheme or in [9] for the deterministic version of the Glimm's scheme -, the convergence results are obtained in 1D for systems of non-linear equations with solutions that are supposed close to constant states. The non-linearity of the systems is then crucial in order to get BV estimates since the initial conditions are not assumed to be BV in [5]. It allows to recover a strong convergence result thanks to compactness arguments based on the Helly's theorem. In the present paper we are concerned in a more simple situation so we can focus on more simple mathematical tools. Since the velocity field is constant and uniform, we are dealing with a linear equation so that it is not necessary to assume that the solutions are close to constant states. Moreover, the considered solutions only take two values. Therefore, the convergence between an approximated solution and the exact solution can be examined by estimating the distance between the approximated fronts and the exact fronts. With the Upwind-GRU scheme, the displacement of the approximated front is governed by a random choice and its location is then associated with a stochastic process. Therefore, the proofs of convergence proposed here rely on the estimation of probabilities (or transitional probabilities), which is a different framework than the one used in [5].

From that point of view, the analysis proposed here is close to the analysis carried out in [4] or [10]. In these references, the Upwind (deterministic) scheme is analyzed by the mean of stochastic processes whose average gives the Upwind scheme, then the key point for obtaining the estimation of the convergence rate is the central limit theorem. An important point to be quoted is that, thanks to general results of probability, the analysis in these references is not restricted to uniform cartesian meshes or to uniform and constant velocity fields. The proofs of sections 4.2, 4.3 and 4.4 are based on exact computations and they are therefore affordable for simple situations. The use of general results, as in [4] or [10], has been shortly addressed in section 4.5 and it should be considered in order to extend the present results to more complex situations.

The paper is organized as follows. In Section 2 the whole Upwind-GRU scheme is described. Then, in Section 3 some useful properties of the Upwind-GRU scheme are exhibited. Actually, these first results allow to understand the way the scheme behaves and provide some preliminary results for the next sections. The convergence results of Section 4 are based on these preliminary results. In this section, a proof of convergence for the one-dimensional case (i.e. the one-dimensional counterpart of system (1)) is first proposed. It helps to understand the proof of convergence for the two-dimensional case. Then, the result of convergence established for the two-dimensional case is briefly extended to the three-dimensional case. Eventually, an other proof is proposed for the two-dimensional case. It relies on general results and it is thus much shorter than the proof of Section 4.3 .

\section{The Upwind-GRU scheme}

We recall here the basis of the Upwind-GRU scheme initially proposed in [8]. The latter is a two-step scheme based on the Upwind scheme and on an update that sharpens the approximated solutions. In order to describe this scheme we introduce here some (classical) notations.

For the sake of simplicity, we consider uniform and cartesian meshes composed of square cells. Here again, we insist on the fact that the scheme can be applied to any kind of meshes, as shown in [8]. The spatial domain is then discretized using cells of size $h \times h$, see Fig. 1 for a sketch of the notations. The center of gravity of cell $(i, j)$ is denoted by $\left(X_{i}, Y_{j}\right)$ and we obviously have: $X_{i+1}=X_{i}+h$ and $Y_{j+1}=Y_{j}+h$. The four faces of the cell $(i, j)$ are denoted in a classical manner by the indices: $(i-1 / 2, j)$ for the left face, $(i+1 / 2, j)$ for the right face, $(i, j-1 / 2)$ for the lower face and $(i, j+1 / 2)$ for the upper face.

We recall that the analysis is restricted here to uniform and constant velocity fields. Hence, let us introduce an angle $\theta$ so that we can write the components of the velocity in the form: $U_{x}=U \cos (\theta)$ and $U_{y}=U \sin (\theta)$, 
where $U \geq 0$ is the norm of the velocity field. Obviously, $\theta$ and $U$ are uniform and constant. Moreover, thanks to the symmetry of the considered meshes, we can choose $\theta \in[0, \Pi / 2]$ without any loss of generality. The other cases $\theta \in[\Pi / 2, \Pi], \theta \in[\Pi, 3 \Pi / 2]$ and $\theta \in[3 \Pi / 2,2 \Pi]$ can be treated in the same manner.

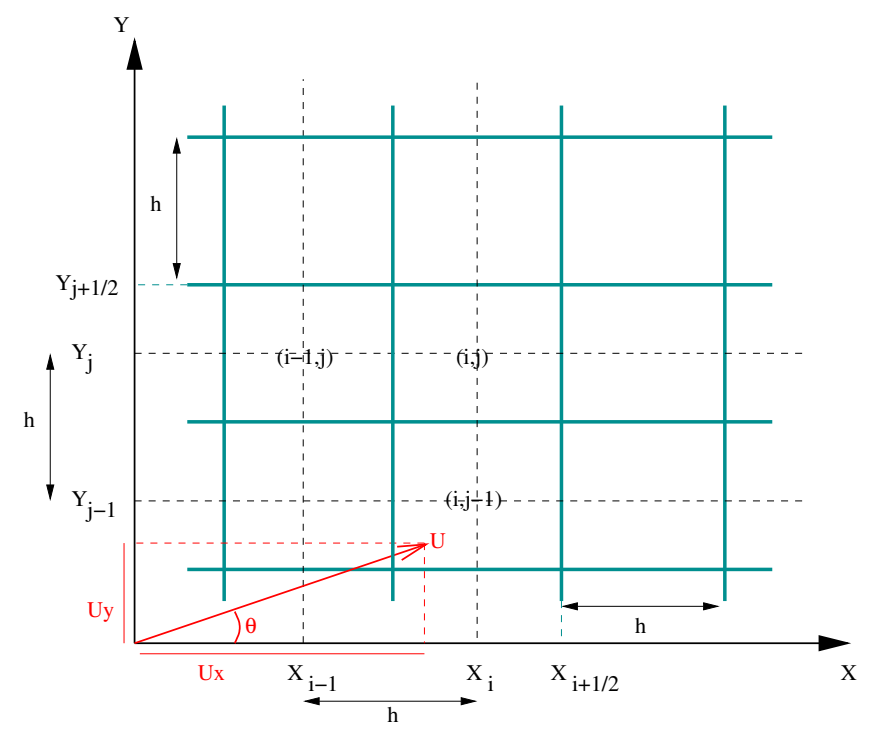

Figure 1: Some notations and definitions for the mesh and the velocity field.

\subsection{First step: update according to the Upwind scheme}

Considering the approximated value of $\Phi$ in cell $(i, j)$ at time $t^{n}$, the Upwind scheme applied to (1) gives the updated value $\Phi_{i, j}^{n+1, *}$ at time $t^{n+1}=t^{n}+\Delta t$ in cell $(i, j)$ :

$$
\Phi_{i, j}^{n+1, *}=\Phi_{i, j}^{n}-\frac{\Delta t}{h}\left(F_{i+1 / 2, j}^{n}-F_{i-1 / 2, j}^{n}\right)-\frac{\Delta t}{h}\left(F_{i, j+1 / 2}^{n}-F_{i, j-1 / 2}^{n}\right) .
$$

The fluxes $F_{i+1 / 2, j}^{n}, F_{i-1 / 2, j}^{n}, F_{i, j+1 / 2}^{n}$ and $F_{i, j-1 / 2}^{n}$ respectively denote the fluxes through the right, left, lower and upper faces of cell $(i, j)$. Due to the specific case we are interested in, and in particular since $\theta \in[0, \Pi / 2]$, we have:

$$
F_{i-1 / 2, j}^{n}=U_{x} \Phi_{i-1, j}^{n}, \quad \text { and } \quad F_{i, j-1 / 2}^{n}=U_{y} \Phi_{i, j-1}^{n} .
$$

Thus, the update formula (2) gives for our specific configurations:

$$
\Phi_{i, j}^{n+1, *}=\Phi_{i, j}^{n}-\frac{U \Delta t}{h}\left(\left(\Phi_{i, j}^{n}-\Phi_{i-1, j}^{n}\right) \cos (\theta)+\left(\Phi_{i, j}^{n}-\Phi_{i, j-1}^{n}\right) \sin (\theta)\right) .
$$

It can thus be seen that the value $\Phi_{i, j}^{n+1, *}$ is a linear combination of the values $\Phi_{i, j}^{n}, \Phi_{i-1, j}^{n}$ and $\Phi_{i, j-1}^{n}$ :

$$
\Phi_{i, j}^{n+1, *}=\Phi_{i-1, j}^{n} \frac{U \Delta t}{h} \cos (\theta)+\Phi_{i, j}^{n}\left(1-\frac{U \Delta t}{h}(\cos (\theta)+\sin (\theta))\right)+\Phi_{i, j-1}^{n} \frac{U \Delta t}{h} \sin (\theta) .
$$

Obviously, equation (4) is a consequence of the choice $\theta \in[0, \Pi / 2]$. Assuming that at time $t^{n}$ the values $\Phi_{i, j}^{n}$ lie in $[0,1]$ for all the cells $(i, j)$, it can be deduced from equation (4) a sufficient CFL condition (for CourantFriedrichs-Lewy condition) which guarantees that $\Phi_{i, j}^{n+1, *}$ also lies in $[0,1]$ in all cells $(i, j)$. Indeed, for a given $\theta \in[0, \Pi / 2]$ and a given velocity $U \geq 0$, if $\Delta t$ and $h$ are such that:

$$
\frac{U \Delta t}{h}(\cos (\theta)+\sin (\theta))<1
$$

then equation (4) corresponds to a convex combination of values belonging to [0,1] and thus $\Phi_{i, j}^{n+1, *}$ also lies in $[0,1]$. In the following, we set $\beta=U \Delta t / h$ and we assume that for a given time step $\Delta t$, the mesh size $h$ is chosen so that $\beta \in\left[0,(\cos (\theta)+\sin (\theta))^{-1}\right]$. For the sake of simplicity, we assume that $\Delta t$ remains the same for all time-iterations. 


\subsection{Second step: the Glimm Random Update}

Let us now assume that the approximated value $\Phi_{i, j}^{n}$ of $\Phi$ in each cell $(i, j)$ at time $t^{n}$ lies in $\{0,1\}$. When applying the Upwind scheme (4) recalled in Section 2.1, the new values $\Phi_{i, j}^{n+1, *}$ generally do not all lie in $\{0,1\}$. The Glimm Random Update proposed in [8] simply consists in updating $\Phi_{i, j}^{n+1, *}$ according to:

$$
\Phi_{i, j}^{n+1}= \begin{cases}1, & \text { if } \omega^{n} \in\left[0, \Phi_{i, j}^{n+1, *}\right] \\ 0, & \text { otherwise }\end{cases}
$$

where $\omega^{n}$ is a random number generated thanks to a uniform distribution on $[0,1]$. A straightforward consequence of this update (5) is that at time $t^{n+1}$ the approximated value $\Phi_{i, j}^{n+1}$ lies in $\{0,1\}$ for all the cells $(i, j)$. It's worth to insist on the fact that $\omega^{n}$ is the same for all the cells, but that $\omega^{n}$ is independent from all the $\omega^{k}$ with $k<n$. It should be noted that this feature was introduced in $[1,2,3]$ considering the Glimm's scheme [5] for the one-dimensional cases. This point is essential for the scheme to behave properly, as shown in the following sections. In particular, this forbids the formation of "holes" in the profiles, as highlighted in the beginning of Section 3.2.

Remark. In the present paper $\omega^{n}$ is a random number generated thanks to a uniform distribution on $[0,1]$. In a practical point of view, $\omega^{n}$ can be replaced by a quasi-random number that is chosen in a low-discrepancy sequence [8]. In [9], the Glimm's scheme associated with a quasi-random number instead of a random number has been examined. According to previous works, the Van Der Corput sequence is one of the most efficient choice, see for instance [3], [7] or [11] (Chapter 5).

\section{Some properties of the Upwind-GRU scheme}

In this section, the scheme presented in Section 2 is translated in terms of stochastic process. Indeed, the complete update of $\Phi_{i, j}^{n}$ into $\Phi_{i, j}^{n+1}$ involves a random choice, so that further analyses of the scheme can be done when considering the approximated values of $\Phi$ as one realization of a stochastic process. In the following, $\mathcal{P}(A)$ denotes the probability of an event $A$ according to the continuous uniform distribution on $[0,1]$, which is the probability distribution for the choice $\omega^{n}$ in the update (5).

\subsection{Expectations and transitional probabilities}

From formula (5) we get the following conditional probabilities knowing the set of the approximated values at time $t^{n}$ and denoted by $\Phi^{n}=\left\{\Phi_{i, j}^{n}: \forall(i, j)\right\}$ :

$$
\mathcal{P}\left(\Phi_{i, j}^{n+1}=1 \mid \Phi^{n}\right)=\Phi_{i, j}^{n+1, *} \quad \text { and } \quad \mathcal{P}\left(\Phi_{i, j}^{n+1}=0 \mid \Phi^{n}\right)=1-\Phi_{i, j}^{n+1, *} .
$$

It should be noted here that it is mandatory to have $0 \leq \Phi_{i, j}^{n+1, *} \leq 1$ so that the two probabilities above are properly defined. This point highlights the importance of the CFL condition of Section 2.1. Considering the probabilities (6), one can easily obtain the conditional expectation $E\left[\Phi_{i, j}^{n+1} \mid \Phi^{n}\right]$ of $\Phi_{i, j}^{n+1} \operatorname{knowing} \Phi_{i, j}^{n}$ :

$$
E\left[\Phi_{i, j}^{n+1} \mid \Phi^{n}\right]=\left(1 \times \mathcal{P}\left(\Phi_{i, j}^{n+1}=1 \mid \Phi^{n}\right)+0 \times \mathcal{P}\left(\Phi_{i, j}^{n+1}=0 \mid \Phi^{n}\right)\right)=\Phi_{i, j}^{n+1, *}
$$

where $\Phi_{i, j}^{n+1, *}$ is given by equation (4). Then, by integrating $E\left[\Phi_{i, j}^{n+1} \mid \Phi^{n}\right]$ over the values of $\Phi^{n}$, the following can be straightforwardly obtained from (4) and (7):

$$
E\left[\Phi_{i, j}^{n+1}\right]=E\left[\Phi_{i-1, j}^{n}\right] \frac{U \Delta t}{h} \cos (\theta)+E\left[\Phi_{i, j}^{n}\right]\left(1-\frac{U \Delta t}{h}(\cos (\theta)+\sin (\theta))\right)+E\left[\Phi_{i, j-1}^{n}\right] \frac{U \Delta t}{h} \sin (\theta) .
$$

It can thus be observed that the expectations $E\left[\Phi_{i, j}^{n}\right]$ are updated according to formula (8), which corresponds to the update formula for the classical Upwind scheme without the GRU step. In that sense the Upwind-GRU scheme corresponds to one way (among others) of selecting one realization of the stochastic process underlying the classical Upwind scheme, see [4].

The update formula (4) implies that the value $\Phi_{i, j}^{n+1, *}$ only depends on values $\Phi_{i, j}^{n}, \Phi_{i-1, j}^{n}$ and $\Phi_{i, j-1}^{n}$. Obviously, combining (4) and (5), we have:

$$
\Phi_{i, j}^{n}=\Phi_{i-1, j}^{n}=\Phi_{i, j-1}^{n} \Longrightarrow \Phi_{i, j}^{n+1}=\Phi_{i, j}^{n} .
$$

Hence, the value in cell $(i, j)$ can change from $t^{n}$ to $t^{n+1}$, i.e. $\Phi_{i, j}^{n+1} \neq \Phi_{i, j}^{n}$, but only when the value of the approximated solution in at least one of its neighboring cells $(i-1, j)$ and $(i, j-1)$ differs from $\Phi_{i, j}^{n}$. This 


\begin{tabular}{|c|c|c|}
\hline$\Phi_{i, j}^{n}=0$ & $\Phi_{i-1, j}^{n}=0$ & $\Phi_{i-1, j}^{n}=1$ \\
\hline$\Phi_{i, j-1}^{n}=0$ & $\Phi_{i, j}^{n+1, *}=0$ & $\Phi_{i, j}^{n+1, *}=\beta \cos (\theta)$ \\
\hline$\Phi_{i, j-1}^{n}=1$ & $\Phi_{i, j}^{n+1, *}=\beta \sin (\theta)$ & $\Phi_{i, j}^{n+1, *}=\beta(\cos (\theta)+\sin (\theta))$ \\
\hline
\end{tabular}

Table 1: Updated value $\Phi_{i, j}^{n+1, *}$ when $\Phi_{i, j}^{n}=0$ with respect to the value in the neighboring cells $(i-1, j)$ and $(i, j-1)$.

\begin{tabular}{|c|c|c|}
\hline$\Phi_{i, j}^{n}=1$ & $\Phi_{i-1, j}^{n}=0$ & $\Phi_{i-1, j}^{n}=1$ \\
\hline$\Phi_{i, j-1}^{n}=0$ & $\Phi_{i, j}^{n+1, *}=1-\beta(\cos (\theta)+\sin (\theta))$ & $\Phi_{i, j}^{n+1, *}=1-\beta \sin (\theta)$ \\
\hline$\Phi_{i, j-1}^{n}=1$ & $\Phi_{i, j}^{n+1, *}=1-\beta \cos (\theta)$ & $\Phi_{i, j}^{n+1, *}=1$ \\
\hline
\end{tabular}

Table 2: Updated value $\Phi_{i, j}^{n+1, *}$ when $\Phi_{i, j}^{n}=1$ with respect to the value in the neighboring cells $(i-1, j)$ and $(i, j-1)$.

implies that during one time-step, the approximated solution only changes in the cells that have neighboring cells which contain a different value at time $t^{n}$. An other consequence is that no "hole" is created by the scheme.

The different possible configurations due to our specific choices are gathered in tables 1 and 2 . From these different cases and according to (5), we can give the following conditional probabilities that represent the transitional probabilities for the stochastic process associated with the Upwind-GRU scheme:

$$
\begin{array}{l|l}
\mathcal{P}\left(\Phi_{i, j}^{n+1}=1\right. & \left.\Phi_{i, j}^{n}=0, \Phi_{i-1, j}^{n}=0, \Phi_{i, j-1}^{n}=0\right)=0, \\
\mathcal{P}\left(\Phi_{i, j}^{n+1}=1\right. & \left.\Phi_{i, j}^{n}=0, \Phi_{i-1, j}^{n}=1, \Phi_{i, j-1}^{n}=0\right)=\beta \cos (\theta), \\
\mathcal{P}\left(\Phi_{i, j}^{n+1}=1\right. & \left.\Phi_{i, j}^{n}=0, \Phi_{i-1, j}^{n}=0, \Phi_{i, j-1}^{n}=1\right)=\beta \sin (\theta), \\
\mathcal{P}\left(\Phi_{i, j}^{n+1}=1\right. & \left.\Phi_{i, j}^{n}=0, \Phi_{i-1, j}^{n}=1, \Phi_{i, j-1}^{n}=1\right)=\beta(\cos (\theta)+\sin (\theta)), \\
\mathcal{P}\left(\Phi_{i, j}^{n+1}=1\right. & \left.\Phi_{i, j}^{n}=1, \Phi_{i-1, j}^{n}=0, \Phi_{i, j-1}^{n}=0\right)=1-\beta(\cos (\theta)+\sin (\theta)), \\
\mathcal{P}\left(\Phi_{i, j}^{n+1}=1\right. & \left.\Phi_{i, j}^{n}=1, \Phi_{i-1, j}^{n}=1, \Phi_{i, j-1}^{n}=0\right)=1-\beta \sin (\theta), \\
\mathcal{P}\left(\Phi_{i, j}^{n+1}=1\right. & \left.\Phi_{i, j}^{n}=1, \Phi_{i-1, j}^{n}=0, \Phi_{i, j-1}^{n}=1\right)=1-\beta \cos (\theta), \\
\mathcal{P}\left(\Phi_{i, j}^{n+1}=1\right. & \left.\Phi_{i, j}^{n}=1, \Phi_{i-1, j}^{n}=1, \Phi_{i, j-1}^{n}=1\right)=1 .
\end{array}
$$

In the relations above, $\mathcal{P}\left(\Phi_{i, j}^{n+1}=1 \mid \Phi_{i, j}^{n}, \Phi_{i-1, j}^{n}, \Phi_{i, j-1}^{n}\right)$ represents the probability to obtain $\Phi_{i, j}^{n+1}=1$, knowing values $\Phi_{i, j}^{n}, \Phi_{i-1, j}^{n}$ and $\Phi_{i, j-1}^{n}$. Obviously, for any of these possible configurations we have:

$$
\mathcal{P}\left(\Phi_{i, j}^{n+1}=0 \quad \mid \quad \Phi_{i, j}^{n}, \Phi_{i-1, j}^{n}, \Phi_{i, j-1}^{n}\right)=1-\mathcal{P}\left(\Phi_{i, j}^{n+1}=1 \quad \mid \quad \Phi_{i, j}^{n}, \Phi_{i-1, j}^{n}, \Phi_{i, j-1}^{n}\right) .
$$

\subsection{Pathological cases}

In this section, we aim at highlighting a pathological behavior of the Upwind-GRU scheme. When considering initial conditions with "thin" shapes on a given mesh (for instance based on a rectangular function in one dimension), the "thin" shape - and thus the fronts - may completely disappear after a finite number of iterations. This point has been highlighted in a numerical point of view in [8]. It will be shown here that mesh refinement tends to decrease the probability of such a behavior for a given final simulation time. Obviously, in a practical point of view, this solution can not always be used and restart strategies could be an alternative.

In order to illustrate this pathological behavior, a "one-dimensional" situation is considered here. Let us choose $\theta=0$, so that the CFL constraint becomes $\beta \in[0,1]$. Let us also assume that at time $t^{n}$ we have:

$$
\Phi_{i, j}^{n}= \begin{cases}1 & \text { if } I_{a} \leq i<I_{b} \\ 0 & \text { otherwise }\end{cases}
$$

with $I_{a}<I_{b}$. We denote by $\Delta I^{N}$ the number of cells in the $x$-direction for which $\Phi_{i, j}^{N}=1$ at iteration $N$, and we have $\Delta I^{n}=I_{b}-I_{a}$. For this very specific case, the CFL condition of Section 2.1 implies that $\beta \in[0,1]$. Thererofe, we assume here that $h$ is fixed and that $\Delta t$ is chosen so that $\beta$ lies in $[1 / 2,1]$.

Following the results of tables 1 and 2 of Section 3.1, we get that after the first step of the Upwind-GRU scheme:

$$
\Phi_{i, j}^{n+1, *}=\left\{\begin{array}{l}
\Phi_{i, j}^{n} \text { if } i \notin\left\{I_{a}, I_{b}\right\} \\
1-\beta \text { if } i=I_{a} \\
\beta \text { if } i=I_{b} .
\end{array}\right.
$$

It can be noticed here that for all cells such that $i \notin\left\{I_{a}, I_{b}\right\}, \Phi_{i, j}^{n+1, *}=\Phi_{i, j}^{n} \in\{0,1\}$. As a consequence we also get that $\Phi_{i, j}^{n+1}=\Phi_{i, j}^{n}$ if $i \notin\left\{I_{a}, I_{b}\right\}$, which shows that no hole is created by the scheme in the initial profile. Moreover, since $\omega^{n}$ is the same for all the cells, the update (9) combined with the step (5) implies that the 
values $\Phi_{i, j}^{n+1}$ at time $t^{n+1}$ do not depend on $j: \forall i, \Phi_{i, j_{0}}^{n+1}=\Phi_{i, j_{1}}^{n+1}$. These remarks imply that $\Delta I^{n}$ can been defined in a unique manner at each iteration.

When turning to cells $i \in\left\{I_{a}, I_{b}\right\}$, according to the second step (5), we have three cases. Since we have chosen $\beta>1 / 2$, we have $0 \leq 1-\beta<\beta \leq 1$ and these cases are the following:

- if $\omega^{n} \in[0,1-\beta]$, we have $\Phi_{I_{a}, j}^{n+1}=1=\Phi_{I_{a}, j}^{n}$ and $\Phi_{I_{b}, j}^{n+1}=1 \neq \Phi_{I_{b}, j}^{n}$, and hence $\Delta I^{n+1}=\Delta I^{n}+1$;

- if $\left.\left.\omega^{n} \in\right] 1-\beta, \beta\right]$, we have $\Phi_{I_{a}, j}^{n+1}=0 \neq \Phi_{I_{a}, j}^{n}$ and $\Phi_{I_{b}, j}^{n+1}=1 \neq \Phi_{I_{b}, j}^{n}$, and hence $\Delta I^{n+1}=\Delta I^{n}$;

- if $\left.\left.\omega^{n} \in\right] \beta, 1\right]$, we have $\Phi_{I_{a}, j}^{n+1}=0 \neq \Phi_{I_{a}, j}^{n}$ and $\Phi_{I_{b}, j}^{n+1}=0=\Phi_{I_{b}, j}^{n}$, and hence $\Delta I^{n+1}=\Delta I^{n}-1$.

When focusing on the width $\Delta I^{n+1}$ of the approximated solution, due to the uniform distribution for $\omega^{n}$, we have the following transitional probabilities :

$$
\begin{array}{r}
\mathcal{P}\left(\Delta I^{n+1}=\Delta I^{n}-1\right)=1-\beta, \\
\mathcal{P}\left(\Delta I^{n+1}=\Delta I^{n}\right)=2 \beta-1, \\
\mathcal{P}\left(\Delta I^{n+1}=\Delta I^{n}+1\right)=1-\beta .
\end{array}
$$

If we have $I_{b}=I_{a}+1$, the probability to set the all cells to 0 is then equal to $(1-\beta)$. Once all the cells are equal to zero, they will remain to zero and the initial profile is definitively lost. The probabilities (10)-(12) clearly advocate for the use of large values of $\beta$.

A more general result can be obtained. Indeed, the parameter $\beta$ is constant and uniform, and the transitional probabilities (10)-(12) thus do not depend on the time-iteration. The random variable $\left(\Delta I^{n+1}-\Delta I^{n}\right)$ is thus associated with a generalized Bernoulli law. Therefore, the random variable $\left(\Delta I^{n}\right)$ follows a multinomial law and each possible realization of a sequence of $n$ iterations of the GRU step is a combination of the three cases mentioned above. Since the probabilities (10)-(12) do not depend on the time-iteration, the probability of one realization for $\Delta I^{n}$ only depends on: the number $I_{-}$of cases (10), the number $I_{0}$ of cases (11) and the number $I_{+}$of cases (12), with $n=I^{-}+I^{0}+I^{+}$. The order of occurrence of the cases does not change the probability. We thus have:

$$
\mathcal{P}\left(I_{-}, I_{0}, I_{+}\right)=C_{I_{-}+I_{0}+I_{+}}^{I_{-}, I_{+}}(1-\beta)^{I_{-}+I_{+}}(2 \beta-1)^{I_{0}},
$$

where $C_{n}^{p, k}$ stands for the number of combinations:

$$
C_{n}^{p, k}=\frac{n !}{p ! k !(n-p-k) !} .
$$

Using these remarks, and starting with a width $\Delta I^{n} \geq 1$ at time $t^{n}$, we can write the probability to set all the values to zero after $p$ iterations. First, if $p<\Delta I^{n}$ it is not possible to set all the cells to zero. For the configurations with $p \geq \Delta I^{n}$, we have to sum the probability of all triplet $\left(I_{-}, I_{0}, I_{+}\right) \in \Delta_{n}^{p}$, where:

$$
\Delta_{n}^{p}=\left\{\left(I_{-}, I_{0}, I_{+}\right) \in \llbracket 0, p \rrbracket^{3} \text { with } I_{-}+I_{0}+I_{+}=p \text { and } I_{-}+I_{+} \geq \Delta I^{n}\right\},
$$

so that we get:

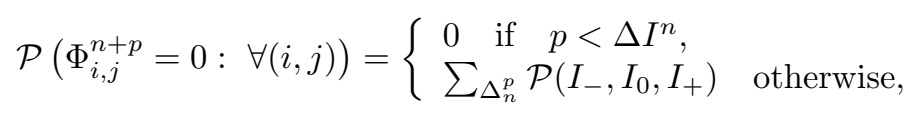

The "worst case" in terms of simulation could be defined as the case with $p=\Delta I^{n}$ and $I_{-}=\Delta I^{n}$. This case corresponds to the minimal number of iterations required to set all the values in cells to zero. It occurs with the probability $(1-\beta)^{\Delta I^{n}}$.

The same kind of results can obviously be obtained for $\beta \in[0,1 / 2]$. Moreover, the cases where all the cells are set to one (instead of zero) can be treated following the same idea. The main point to notice here is that for coarse meshes, some parts of the initial solution may disappear with a non-zero probability. Nonetheless, for a given physical width of the initial solution and for a given final time, mesh refinement decreases the probability to set all the values in the cells to zero.

\section{A result of convergence for the Upwind-GRU scheme}

In this section, we propose a proof of convergence of the Upwind-GRU scheme when considering the advection of a planar front through system (1). We first begin with the one-dimensional case. Due to our assumption of constant and uniform velocity, the Upwind-GRU scheme exactly coincides with the Glimm scheme in our one-dimensional case. Hence the proof of convergence of the latter is sufficient to prove the convergence of the former. Nevertheless, the proof that we propose in Section 4.3 for the Upwind-GRU scheme in the twodimensional case is an extension of the proof of Section 4.2 for the one-dimensional case. Starting with the 
one-dimensional case may help the reader to grasp the main ingredients of the proof. This section begins by a short subsection that recall some useful and basic notions of probability.

Throughout this section, the mesh described in Section 2 and the corresponding notations are used. The one-dimensional case of Section 4.2 corresponds to $\theta=0$ and, for the sake of readability, notations associated with the $y$ direction are omitted.

\subsection{Notion of convergence in the framework of probability.}

The aim of this subsection is to recall some basic notions for the convergence of sequences of random variables. A classical result issued from the Borel-Cantelli's theorem is also proposed. It is indeed very useful in order to prove the convergence of a sequence of random variables

Let $\left(X_{n}\right)_{n \geq 0}, X_{n} \in \mathbb{R}$, be a sequence of random variables in a probability space associated with the probability measure $P$. A sequence $\left(X_{n}\right)_{n \geq 0}$ is said to converge in probability towards $X \in \mathbb{R}$ when $n$ tends towards $+\infty$, or in short $X_{n} \stackrel{p}{\rightarrow} X$, when:

$$
\forall \eta>0, \lim _{n \rightarrow+\infty} P\left(\left\{\left|X_{n}-X\right|>\eta\right\}\right)=0 .
$$

A sequence $\left(X_{n}\right)_{n \geq 0}$ is said to converge almost surely towards $X$ when $n$ tends towards $+\infty$, or in short $X_{n} \stackrel{\text { as }}{\rightarrow} X$, when:

$$
\forall \eta>0, P\left(\cap_{k=0}^{+\infty}\left(\cup_{m \geq k}\left\{\left|X_{m}-X\right|>\eta\right\}\right)\right)=0
$$

or in an equivalent manner:

$$
P\left(\left\{\lim _{n \rightarrow+\infty}\left|X_{n}-X\right|=0\right\}\right)=1 .
$$

The convergence almost surely is stronger than the convergence in probability in the sense that:

$$
X_{n} \stackrel{\text { as }}{\rightarrow} X \Longrightarrow X_{n} \stackrel{p}{\rightarrow} X
$$

We can now introduce a result issued from the Borel-Cantelli's theorem, and that is commonly used in order to prove the convergence almost surely of a sequence $\left(X_{n}\right)_{n \geq 0}$ towards $X$. Let us assume that for all $\eta>0$, the sequence $\left(\sum_{n=0}^{N} P\left(\left\{\left|X_{n}-X\right|>\eta\right\}\right)\right)_{N \geq 0}$ is convergent, which is equivalent to:

$$
\lim _{N \rightarrow+\infty} \sum_{n=N}^{+\infty} P\left(\left\{\left|X_{n}-X\right|>\eta\right\}\right)=0, \quad \text { or } \quad \exists C>0, \lim _{N \rightarrow+\infty} \sum_{n=0}^{N} P\left(\left\{\left|X_{n}-X\right|>\eta\right\}\right) \leq C .
$$

For any $\eta>0$, thanks to the sub-additivity and to the monotony of the measure $P$, we have for all $N$ :

$$
P\left(\cap_{k=0}^{\infty}\left(\cup_{m \geq k}\left\{\left|X_{m}-X\right|>\eta\right\}\right)\right) \leq P\left(\cup_{m \geq N}\left\{\left|X_{m}-X\right|>\eta\right\}\right) \leq \sum_{m=N}^{+\infty} P\left(\left\{\left|X_{m}-X\right|>\eta\right\}\right) .
$$

Hence, taking the limit $N \rightarrow+\infty$ of this inequality leads to:

$$
P\left(\cap_{k=0}^{+\infty}\left(\cup_{m \geq k}\left\{\left|X_{m}-X\right|>\eta\right\}\right)\right)=0,
$$

which corresponds to the definition of the convergence almost surely $X_{n} \stackrel{a s}{\rightarrow} X$. The present result, issued from the Borel-Cantelli's theorem, can be stated as follows:

$$
\forall \eta>0, \lim _{N \rightarrow+\infty} \sum_{n=N}^{+\infty} P\left(\left\{\left|X_{n}-X\right|>\eta\right\}\right)=0 \quad \Longrightarrow \quad X_{n} \stackrel{\text { as }}{\rightarrow} X .
$$

\subsection{One-dimensional case}

For the sake of simplicity, all subscripts $j$ that are related to the coordinate $y$, and the $y$ components and coordinates are omitted. We consider here the one-dimensional counterpart of system of equations (1):

$$
\left\{\begin{array}{l}
\partial_{t} \Phi(t, x)+U \partial_{x} \Phi(t, x)=0 \\
\Phi(t=0, x)=\Phi^{0}(x)
\end{array}\right.
$$

where the velocity $U$ is constant and uniform. The initial condition is:

$$
\Phi^{0}(x)= \begin{cases}1 & \text { if }\left(x-X_{f}^{0}\right)<0 \\ 0 & \text { otherwise }\end{cases}
$$


where $X_{f}^{0}$ stands for a given abscissa. It represents here the initial position of the front. Thanks to our assumptions, the exact solution of system (14) with initial condition (15) can straightforwardly be written as:

$$
\forall t>0, \forall x, \Phi(t, x)=\Phi^{0}(x-U t) .
$$

The first point here is to choose an approximated initial condition for the numerical scheme. Let us take the classical approximated initial solution which is equal to the value of the initial condition (15) at the center of gravity of the cell, that is:

$$
\Phi_{i}^{0}=\Phi^{0}\left(X_{i}\right)
$$

It is an important point to be quoted that we obviously have:

$$
\sum_{i} \int_{X_{i-1 / 2}}^{X_{i+1 / 2}}\left|\Phi^{0}(x)-\Phi_{i}^{0}\right| d x=O(h) .
$$

Remark. Approximated initial conditions have been chosen here according to (17). It should be noted that this choice has no consequence on the results of this section. The only requirement herein is to choose approximated initial conditions that fulfill (18).

Starting with this approximated initial solution, the Upwind-GRU scheme defined in Section 2 is then applied for a given time-step $\Delta t$ such that the CFL condition of Section 2.1 is fulfilled. The following proposition then holds.

Proposition 4.1 With an approximated initial solution of the form (15) and (17) at time $t^{0}=0$, the transitional probabilities for the approximated solution from time $t^{n}$ to time $t^{n+1}=t^{n}+\Delta t$, obtained with the Upwind-GRU scheme are:

$$
\left\{\begin{array}{l}
\mathcal{P}\left(\forall i, \Phi_{i}^{n+1}=\Phi_{i-1}^{n}\right)=\beta, \\
\mathcal{P}\left(\forall i, \Phi_{i}^{n+1}=\Phi_{i}^{n}\right)=1-\beta .
\end{array}\right.
$$

The parameter $\beta$ corresponds to the CFL number and it must belong to ]0,1[ in order to ensure the stability of the numerical scheme.

Proof. Due to our choices for the velocity field and the initial condition there are only three possibilities among the configurations gathered in tables 1 and 2 :

- case 1: $\Phi_{i}^{0}=0$ and $\Phi_{i-1}^{0}=1$;

- case 2: $\Phi_{i}^{0}=0$ and $\Phi_{i-1}^{0}=0$;

- case 3: $\Phi_{i}^{0}=1$ and $\Phi_{i-1}^{0}=1$.

For any cell $i$ in the case 2 or 3 , updating the approximated solution through the Upwind-GRU scheme does not change the value in the cell: $\Phi_{i}^{1}=\Phi_{i}^{0}$. Indeed, the update through the Upwind-GRU scheme implies that a change may only occur in a cell that is in case 1 . Considering the specific form of the initial condition in this section, there is a unique cell $i_{0}$ in case 1 at first iteration. We have in that cell the transitional probability:

$$
\mathcal{P}\left(\Phi_{i_{0}}^{1}=1 \quad \mid \quad \Phi_{i_{0}}^{0}=0, \Phi_{i_{0}-1}^{0}=1\right)=\beta
$$

Since the values in the cells that are in case 2 or 3 remain unchanged with probability 1 , the update of the approximated solution $\Phi^{1}$ corresponds to a translation of the initial approximated solution $\Phi^{0}$ :

- from $+h$ to the right with respect to the positive $x$ when $\Phi_{i_{0}}^{1}=1$, in other words $\forall i, \Phi_{i}^{1}=\Phi_{i-1}^{0}$;

- from 0 when $\Phi_{i_{0}}^{1}=0$, which means that $\Phi^{1}=\Phi^{0}$.

Thus, at the end of the first iteration there is always a unique cell which is in case 1, and all the other cells are in case 2 or 3 . Moreover, we then obtain the following transitional probabilities:

$$
\left\{\begin{array}{l}
\mathcal{P}\left(\forall i, \Phi_{i}^{1}=\Phi_{i-1}^{0}\right)=\beta, \\
\mathcal{P}\left(\forall i, \Phi_{i}^{1}=\Phi_{i}^{0}\right)=1-\beta .
\end{array}\right.
$$

It should be noted that the transitional probabilities (20) do not depend on the time iteration (we recall that $\beta$ is fixed in our specific case), and since the initial profile is just translated from $h$ or 0 , we deduce that the probabilities (20) remain the same for all the iterations. We thus get the transitional probabilities (19) which hold for any iteration time $t^{n}$. This ends the proof of proposition 4.1 .

Let us consider $N>0$ time-iterations. As mentioned above in the proof of proposition 4.1, each realization of the Upwind-GRU scheme for $N$ iterations is thus composed of $K \in \llbracket 0, N \rrbracket$ shifts of $h$ to the right of the approximated initial solution $\Phi^{0}$ and $N-K$ standstills. The probability of each configuration is given by proposition 4.2. 
Proposition 4.2 The probability $P_{N}^{K}$ to get exactly $K \in \llbracket 0, N \rrbracket$ shifts for $N$ iterations with the Upwind-GRU scheme is:

$$
P_{N}^{K}=C_{N}^{K}(\beta)^{K}(1-\beta)^{N-K}
$$

where $C_{N}^{K}$ stands for the number of combinations:

$$
C_{N}^{K}=\frac{N !}{K !(N-K) !}
$$

Proof. As mentioned above in the proof of proposition 4.1, for each iteration we have the same transitional probabilities (19). Moreover, the random numbers $\omega^{n}$ involved in the second step of the Upwind-GRU scheme are independent for all the iterations. Hence each realization is independent and follows (19). Therefore, all the configurations of $K$ shifts and $N-K$ standstills are equivalent in terms of probability. As a consequence, the configurations obtained after $N$ iterations with the Upwind-GRU scheme follow the binomial distribution with the parameter $\beta: \mathcal{B}(N, \beta)$. Thus, we obtain the formula $(21)$ for the probability $P_{N}^{K}$ to get $K$ shifts for $N$ iterations.

Hence, the configurations obtained with the Upwind-GRU scheme follow the binomial distribution $\mathcal{B}(N, \beta)$. Since the displacement associated with each configuration is known, we can deduce $E_{d}^{N}$ the expectation of the displacement of the approximated initial solution after $N$ iterations.

Proposition 4.3 The expectation of the displacement of the approximated initial solution after $N$ iterations $E_{d}^{N}$ is equal to the displacement of the approximated initial solution through system (14).

Proof. For each configuration with $N$ iterations and exactly $K$ shifts, the displacement of the approximated initial solution is equal to $K h$. We then get from proposition 4.2 that $E_{d}^{N}$ is:

$$
\begin{array}{r}
E_{d}^{N}=\sum_{K=0}^{N}\left(P_{N}^{K} K h\right), \\
E_{d}^{N}=h \sum_{K=1}^{N}\left(K C_{N}^{K}(\beta)^{K}(1-\beta)^{N-K}\right), \\
E_{d}^{N}=h \sum_{K=1}^{N}\left(N C_{N-1}^{K-1}(\beta)^{K}(1-\beta)^{N-K}\right), \\
E_{d}^{N}=N \beta h \sum_{K=0}^{N-1}\left(C_{N-1}^{K}(\beta)^{K}(1-\beta)^{(N-1)-K}\right) .
\end{array}
$$

The sum on the right hand side of equation $(25)$ is the development of the polynomial $(\beta+(1-\beta))^{N-1}$ which is obviously equals to 1 . We thus finally get:

$$
E_{d}^{N}=N \beta h
$$

By introducing the definition of $\beta=U \Delta t / h$, we can write:

$$
E_{d}^{N}=N \beta h=U N \Delta t=U t^{N}
$$

The displacement $U t^{N}$ corresponds exactly to the displacement of the approximated initial solution between $t=0$ and $t=t^{N}$ when it is considered as the initial condition for system (14). This ends the proof of proposition 4.3 .

Thanks to proposition 4.3, we know that the Upwind-GRU scheme implies an exact average displacement of the approximated initial solution. The proof proposed in this section is based on the fact that the probability distribution of one realization of the displacement for a given $N$, based on (21), corresponds to a distribution that is centered on a neighborhood of the displacement $E_{d}^{N}$. By studying the probabilities $P_{N}^{K}$ given by $(21)$, we obtain proposition (4.4).

Proposition 4.4 For any number of iterations $N>0$, there exists an integer $0 \leq K^{N, \beta}<N$, such that $K \mapsto P_{N}^{K}$ increases for $K \leq K^{N, \beta}$ and decreases for $K>K^{N, \beta}$. Therefore, the probability $P_{N}^{K}$ reaches a maximum for $K=K^{N, \beta}$ or $K=K^{N, \beta}+1$. Moreover, the displacement associated with $K^{N, \beta}$ fulfills:

$$
\left|K^{N, \beta} h-E_{d}^{N}\right|<2 h
$$


Proof. For a given number of iterations $N$, simple calculus rules lead to the relation:

$$
P_{N}^{K+1}=P_{N}^{K} \frac{N-K}{K+1} \frac{\beta}{1-\beta} .
$$

Hence we get that

$$
P_{N}^{K+1} \geq P_{N}^{K} \Longleftrightarrow K \leq \beta N-(1-\beta) .
$$

We denote $K^{N, \beta}$ the integer part of $(\beta N-(1-\beta))$, we then have:

$$
P_{N}^{K+1} \geq P_{N}^{K} \Longleftrightarrow K \leq K^{N, \beta} .
$$

From the definition of $K^{N, \beta}$, since $0<\beta<1$ and thanks to relation (26) from proposition 4.3, it yields:

$$
\left|K^{N, \beta} h-E_{d}^{N}\right|<2 h .
$$

Hence, from (29) and (28), we deduce that $K \mapsto P_{N}^{K}$ increases for $K \leq K^{N, \beta}$ and decreases for $K>K^{N, \beta}$. Therefore, the probability $P_{N}^{K}$ reaches a maximum for $K=K^{N, \beta}$ or $K=K^{N, \beta}+1$. This ends the proof for proposition 4.4.

As an illustration of proposition 4.4, the probabilities $P_{N}^{K}$ are plotted on Fig. 2 with respect to $K / N$ for $N=100$ and $\beta=0.75$. In this case, we have $K^{100,0.75}=74$ and the maximum is reached for $K=75$. It can be observed that the probabilities $P_{N}^{K}$ quickly tend to zero as $K$ deviates from $K^{100,0.75}$. This feature also plays an important role in the present proof, and in particular for proposition 4.5.

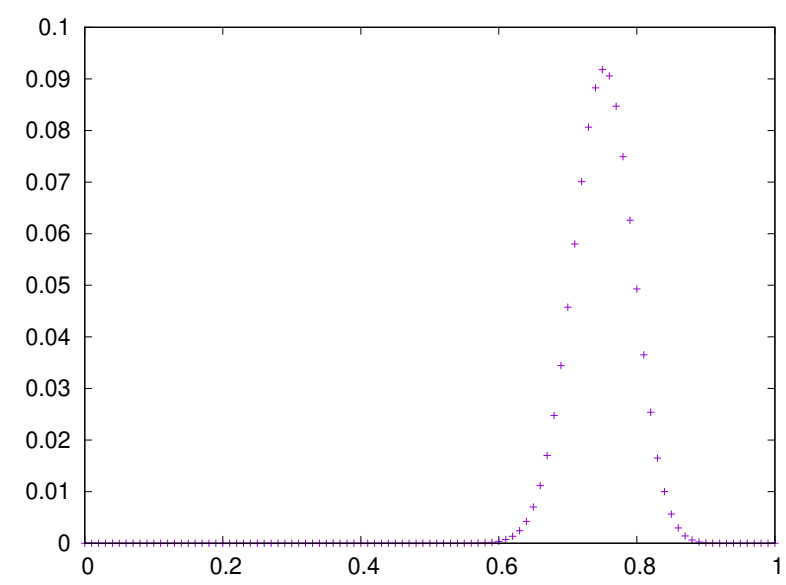

Figure 2: Probabilities $P_{N}^{K}$ ( $y$-axis) with respect to $K / N$ ( $x$-axis) for $N=100$ and $\beta=0.75$. The maximum is reached for $K=75$ (with $K^{100,0.75}=74$ ) and its value is $P_{100}^{75} \sim 0.0917997$.

Let us fix a final time $T$ and a CFL number $\beta$. The time-step is thus deduced from $T$ and from the number of iterations $N$ through: $\Delta t=T / N$; hence, the mesh size is obtained from the CFL condition: $h=T U /(N \beta)$. For a given $\eta>0$, the probability for the displacement of the approximated initial solution to be in the interval $\left[E_{d}^{N}-\eta, E_{d}^{N}+\eta\right]$ after $N$ time-iterations is denoted by $\mathcal{P}_{N}^{\eta}$. We can state the following proposition for $\mathcal{P}_{N}^{\eta}$.

Proposition 4.5 Let us assume that the final time $T$ and the $C F L$ number $\beta \in] 0,1[$ are given. Let $N$ be the total number of time-steps for reaching the final time $T$, so that both $h$ and $\Delta t$ are known for any number of iterations $N$. Then, the displacement of the approximated initial solution by the Upwind-GRU scheme after $N$ time-iterations converges almost surely to the exact displacement $E_{d}^{N}=T U$ when $N$ tends towards $+\infty$ :

$$
\mathcal{K}^{N} h \stackrel{a s}{\longrightarrow} T U
$$

where the random variable $\left(\mathcal{K}^{N}\right)$ stands for the random variable associated with the number of shifts obtained by the Upwind-GRU scheme for $N$ iterations.

Proof. Let us denote by $\mathcal{P}_{N}^{\eta}$ the probability for the displacement of the approximated initial solution to be in the interval $\left[E_{d}^{N}-\eta, E_{d}^{N}+\eta\right]$ after $N$ time-iterations. The probability $\mathcal{P}_{N}^{\eta}$ is defined as:

$$
\mathcal{P}_{N}^{\eta}=\sum_{K \in \mathcal{H}_{N}(\eta)} P_{N}^{K}
$$


where $\mathcal{H}_{N}(\eta)=\left\{K \in \llbracket 0, N \rrbracket,\left|E_{d}^{N}-K h\right| \leq \eta\right\}$ and $E_{d}^{N}=T U$. So, thanks to relation (28), there exists a number of iterations $N_{\eta}$ for which we have:

$$
\forall N \geq N_{\eta},\left\{K^{N, \beta}, K^{N, \beta}+1\right\} \subset \mathcal{H}_{N}(\eta) .
$$

From now on we assume that $N$ is greater than $N_{\eta}$. Let us also define $K_{\text {inf }}$ (resp. $K_{\text {sup }}$ ) as the integer part of $\left(E_{d}^{N}-\eta\right) / h+1$ (resp. of $\left.\left(E_{d}^{N}+\eta\right) / h-1\right)$. Obviously, $K_{\text {inf }}$ and $K_{\text {sup }}$ belong to $\mathcal{H}_{N}(\eta)$. However, since we have chosen $N>N_{\eta}$ and thanks to (29), we have the relation:

$$
\forall K \notin \mathcal{H}_{N}(\eta), P_{N}^{K}<\max \left(P_{N}^{K_{\text {inf }}}, P_{N}^{K_{\text {sup }}}\right)
$$

from which we get that:

$$
1-\mathcal{P}_{N}^{\eta}=\sum_{K \notin \mathcal{H}_{N}(\eta)} P_{N}^{K} \leq \operatorname{card}\left(\mathcal{H}_{N}^{\prime}(\eta)\right) \times \max \left(P_{N}^{K_{\text {inf }}}, P_{N}^{K_{\text {sup }}}\right) \leq N \max \left(P_{N}^{K_{\text {inf }}}, P_{N}^{K_{\text {sup }}}\right)
$$

where $\mathcal{H}_{N}^{\prime}(\eta)$ is the complementary set of $\mathcal{H}_{N}(\eta): \mathcal{H}_{N}^{\prime}(\eta)=\left\{K \in \llbracket 0, N \rrbracket,\left|E_{d}^{N}-K h\right|>\eta\right\}$. Thanks to our choices, we have in fact:

$$
\left(E_{d}^{N}-\eta\right) / h+1=N \beta\left(1-\frac{\eta}{T U}\right)+1 \quad \text { and } \quad\left(E_{d}^{N}+\eta\right) / h-1=N \beta\left(1+\frac{\eta}{T U}\right)-1 .
$$

It means that for $0<\eta<T U, K_{\text {inf }}$ and $K_{\text {sup }}$ tend to $+\infty$ as fast as $N$ when $N$ tends to $+\infty$. Since in the definition of $P_{N}^{K}$, the integer $K$ is the exponent of $\left.\beta \in\right] 0,1[$, it means that the term involving the maximum on the right hand side of (30) tends to zero faster than $1 / N$ when $N$ tends towards $+\infty$. As a consequence, we get that:

$$
0 \leq 1-\mathcal{P}_{N}^{\eta} \leq N \max \left(P_{N}^{K_{\text {inf }}}, P_{N}^{K_{\text {sup }}}\right) \underset{N \rightarrow+\infty}{\longrightarrow} 0
$$

whereof we deduce that the probability for the displacement of the approximated initial solution to be at a distance less than $\eta$ from the exact displacement tends to 1 when the number of iterations tends towards $+\infty$. Moreover, by summing inequality (31) for $N=1$ to $N=N_{0} \geq 1$ we get:

$$
0 \leq 1-\sum_{N=1}^{N_{0}}\left(\mathcal{P}_{N}^{\eta}\right) \leq \sum_{N=1}^{N_{0}}\left(N \max \left(P_{N}^{K_{\text {inf }}}, P_{N}^{K_{\text {sup }}}\right)\right) .
$$

Since in the definition of $P_{N}^{K}$, the integer $K$ is the exponent of $\left.\beta \in\right] 0,1[$, the sum on the right and side of inequality (32) remains bounded when $N_{0}$ tends towards $+\infty$. So that for all $\eta>0$, there exists a constant $C_{1}>0$ such that:

$$
\sum_{N=1}^{\infty}\left(P\left(\left|E_{d}^{N}-\mathcal{K}^{N} h\right| \geq \eta\right)\right) \leq C_{1}
$$

Thanks to this bound, the Borel-Cantelli's theorem then states that the random variable associated with the approximated displacement $\left(\mathcal{K}^{N} h\right)$ converges almost surely to the exact displacement $E_{d}^{N}=T U$ when the number of iterations tends towards $+\infty$. This ends the proof the proposition 4.5.

Proposition 4.5 proves that the displacements of the approximated initial solution due to the Upwind-GRU scheme tend almost surely towards the exact displacement when the number of iterations $N$ tends towards $+\infty$. We can then conclude the whole proof with the following theorem which mainly relies in the results of proposition 4.5.

Theorem 4.1 Let us assume that the final time $T$ and the $C F L$ number $\beta \in] 0,1[$ are given. Let $N$ be the total number of time-steps for reaching the final time $T$, so that both $h$ and $\Delta t$ are known for any number of iterations $N$. The approximated solutions computed with the Upwind-GRU scheme at time T for a CFL number $\beta$ converge almost surely towards the exact solution when $N$ tends towards $+\infty$ :

$$
X_{f}^{N, g r u} \stackrel{a s}{\longrightarrow} X_{f}(T),
$$

where $X_{f}(T)$ is the position of the exact front at time $T$, and $X_{f}^{N, g r u}$ the position of the front for the approximated solution at time $T$ for $N$ iterations.

Proof. For a final time $T$ and a CFL number $\beta \in] 0,1[$, both $h$ and $\Delta t$ are known for any number of iterations $N$ through the relations: $\Delta t=T / N$ and $h=T U /(N \beta)$. The position of the approximated initial front $X_{f}^{0, N}$ 
for the approximated initial condition (17) also only depends on the number of iterations $N$. When considering the initial condition associated with (17):

$$
\begin{cases}1 & \text { if }\left(x-X_{f}^{0, N}\right)<0 \\ 0 & \text { otherwise }\end{cases}
$$

the position $X_{f}^{N}(T)$ of the front at time $T$ of the exact solution of system (1) with initial condition (33) is:

$$
X_{f}^{N}(T)=X_{f}^{0, N}+U T
$$

Let us denote by $X_{f}(T)$ the position at time $T$ of the exact front in the solution (16) of system of equations (1) for the initial condition (15):

$$
X_{f}(T)=X_{f}^{0}+U T
$$

We know from proposition 4.1 that for the approximated initial condition (17), the approximated solution computed by the Upwind-GRU corresponds to a translation of the approximated initial condition. We can thus define a front location $X_{f}^{N, g r u}$ for the approximated solution computed by the Upwind-GRU scheme after $N$ iterations.

By considering a number of shifts $0 \leq \mathcal{K}^{N} \leq N$, the approximated front is such that:

$$
\left|X_{f}(T)-X_{f}^{N, g r u}\right|=\left|\left(X_{f}^{0}+U T\right)-\left(X_{f}^{0, N}+\mathcal{K}^{N} h\right)\right|=\left|\left(X_{f}^{0}-X_{f}^{0, N}\right)+\left(U T-\mathcal{K}^{N} h\right)\right| .
$$

Thanks to our choice for the approximated initial condition, we obtain that:

$$
\left|X_{f}(T)-X_{f}^{N}(T)\right|=\left|X_{f}^{0, N}-X_{f}^{0}\right| \leq h,
$$

and thus that:

$$
\left|X_{f}(T)-X_{f}^{N, g r u}\right| \leq h+\left|U T-\mathcal{K}^{N} h\right|=\frac{T U}{\beta} \frac{1}{N}+\left|U T-\mathcal{K}^{N} h\right| .
$$

Since $T, U$ and $\beta$ are fixed, when $N$ tends towards $+\infty$, we have $T U /(\beta N) \rightarrow 0$. Moreover, thanks to proposition 4.5 we also have $\left|U T-\mathcal{K}^{N} h\right| \stackrel{a s}{\longrightarrow} 0$. It can therefore be concluded that:

$$
\left|X_{f}(T)-X_{f}^{N, g r u}\right| \stackrel{a s}{\longrightarrow} 0
$$

when $N$ tends towards $+\infty$. This ends the proof of theorem 4.1.

Finally, we have proved that the approximated solutions computed with the Upwind-GRU scheme converge towards the exact solution almost surely for the specific problem involving: a constant and uniform velocity and an initial condition based on the Heaviside function. It is possible to extend this result to more general initial conditions while keeping a constant and uniform velocity. Nevertheless, for these more general cases, it should be accounted for the pathological behavior described in Section 3.2. It should still be noted that in the case of non constant and non-uniform velocities, the proof becomes very tricky. In particular expressing the probabilities (21) as in proposition 4.2 is no longer possible since the transitional probabilities that are used in the proof of proposition 4.1 depend on the time-iteration. This is indeed the key point in the proof proposed here.

\subsection{Two-dimensional case}

In the following, the results of Section 4.2 are extended to the two-dimensional case for $\theta \in[0, \Pi / 4]$ (the other cases can be studied in a similar way). Each proposition of the previous section has thus its counterpart in the present section. Let us begin by defining the initial condition:

$$
\Phi^{0}(x, y)= \begin{cases}1 & \text { if }\left(x-X_{f}^{0}\right) \cos (\theta)+\left(y-Y_{f}^{0}\right) \sin (\theta)<0 \\ 0 & \text { otherwise. }\end{cases}
$$

for system (1). We define the line $D_{f}^{0}:\left(x-X_{f}^{0}\right) \cos (\theta)+\left(y-Y_{f}^{0}\right) \sin (\theta)=0$ where $\left(X_{f}^{0}, Y_{f}^{0}\right)$ corresponds to a reference point of $D_{f}^{0}$, and $n_{0}=(\cos (\theta), \sin (\theta))$ corresponds to the unit vector which is orthogonal to $D_{f}^{0}$ and oriented following the velocity field (we recall that here $\theta \in[0, \Pi / 4]$ ). This initial condition corresponds to $\Phi^{0}(x, y)=1$ for all the points $(x, y)$ below $D_{f}^{0}$ and to $\Phi^{0}(x, y)=0$ for all the points $(x, y)$ above $D_{f}^{0}$. Thanks to the assumptions detailed in Section 2, the exact solution of (1) with the initial condition (34) is:

$$
\Phi(t, x, y)= \begin{cases}1 & \text { if }\left(x-X_{f}(t)\right) \cos (\theta)+\left(y-Y_{f}(t)\right) \sin (\theta)<0 \\ 0 & \text { otherwise }\end{cases}
$$




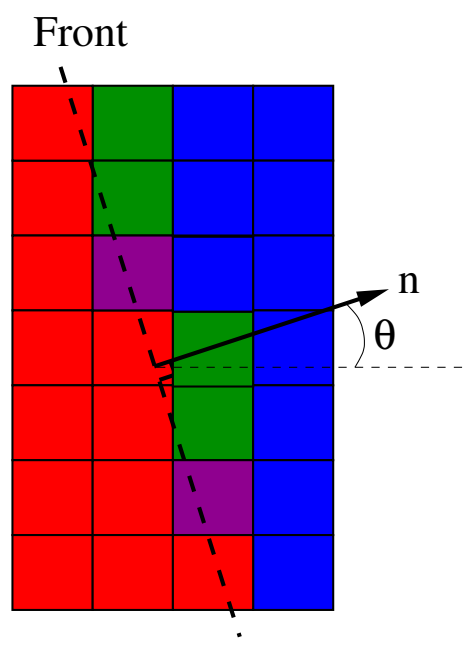

Figure 3: Example of a front on a given mesh at the beginning of a time-iteration. The front is represented by the dashed line, and $n$ stands for its normal vector. The red cells contain the value 1 , and all the other one contain the value 0 . Therefore: red cells correspond to case 4 , blue cells correspond to case 3 , green cells correspond to case 1 and purple cells correspond to case 2

where the reference point $\left(X_{f}(t), Y_{f}(t)\right)$ corresponds to the translation of the reference point $\left(X_{f}^{0}, Y_{f}^{0}\right)$ from the vector $(t U \cos (\theta), t U \sin (\theta))$, that is:

$$
X_{f}(t)=X_{f}^{0}+t U \cos (\theta) \quad \text { and } \quad Y_{f}(t)=Y_{f}^{0}+t U \sin (\theta)
$$

Let us now turn to the approximated solutions of (35) and (36) computed by the Upwind-GRU scheme.

We first define the approximated initial condition $\Phi_{i, j}^{0}$ for the Upwind-GRU scheme. Even if several choices are possible, they have no influence on the results of this section provided that we have:

$$
\sum_{(i, j)} \int_{(i, j)}\left|\Phi(t=0, x, y)-\Phi_{i, j}^{0}\right| d x d y=O(h) .
$$

In the following, we define:

$$
\Phi_{i, j}^{0}=\Phi^{0}\left(X_{i}, Y_{j}\right),
$$

which is a classical choice fulfilling (37). We recall that, according to the definitions of section $2, X_{i}$ and $Y_{j}$ are the coordinates of the center of gravity of cell $(i, j)$ (see also Fig. 1).

Remark. As in Section 4.2, all the approximated initial conditions fulfilling condition (37) can be chosen instead of (38) for obtaining the results of this section.

Starting from this initial condition, we then apply the Upwind-GRU scheme with a time-step $\Delta t$ such that the CFL condition of Section 2.1 is fulfilled. Then the following proposition holds for the transitional probabilities.

Proposition 4.6 With an approximated initial solution of the form (34) and (38) at time $t^{0}=0$, the transitional probabilities for the approximated solution from time $t^{n}$ to time $t^{n+1}=t^{n}+\Delta t$, obtained with the Upwind-GRU scheme are:

$$
\left\{\begin{array}{l}
\mathcal{P}\left(\forall(i, j), \Phi_{i, j}^{n+1}=\Phi_{i-1, j}^{n}\right)=\beta \cos (\theta), \\
\mathcal{P}\left(\forall(i, j), \Phi_{i, j}^{n+1}=\Phi_{i, j-1}^{n}\right)=\beta \sin (\theta), \\
\mathcal{P}\left(\forall(i, j), \Phi_{i, j}^{n+1}=\Phi_{i, j}^{n}\right)=1-\beta(\cos (\theta)+\sin (\theta)) .
\end{array}\right.
$$

The parameter $\beta$ corresponds to the CFL number and it should belong to $] 0,(\cos (\theta)+\sin (\theta))^{-1}[$ in order to ensure the stability of the numerical scheme.

Proof. Due to our choices for the velocity field and the initial condition, we are faced to only four of the eight configurations gathered in tables 1 and 2:

- case 1: $\Phi_{i, j}^{0}=0, \Phi_{i-1, j}^{0}=1$, and $\Phi_{i, j-1}^{0}=0$;

- case 2: $\Phi_{i, j}^{0}=0, \Phi_{i-1, j}^{0}=1$, and $\Phi_{i, j-1}^{0}=1$; 
- case 3: $\Phi_{i, j}^{0}=0, \Phi_{i-1, j}^{0}=0$, and $\Phi_{i, j-1}^{0}=0$;

- case 4: $\Phi_{i, j}^{0}=1, \Phi_{i-1, j}^{0}=1$, and $\Phi_{i, j-1}^{0}=1$.

Cases 3 and 4 correspond to cells $(i, j)$ for which the approximated value of $\Phi$ can not change at the first iteration: $\Phi_{i, j}^{1}=\Phi_{i, j}^{0}$. We thus focus on the cells of cases 1 and 2 , that are the cells just downwind the approximated front, see Fig. 3. For these cells, the first step of the Upwind-GRU scheme leads to the values:

- case $1: \Phi_{i, j}^{1, *}=\beta \cos (\theta)$;

- case 2: $\Phi_{i, j}^{1, *}=\beta(\cos (\theta)+\sin (\theta)$.

Then, the second step of the Upwind-GRU scheme leads to:

- case 1: $\Phi_{i, j}^{1}=1$ if $\omega^{0} \in[0, \beta \cos (\theta)]$, and $\Phi_{i, j}^{1, *}=0$ otherwise;

- case 2: $\Phi_{i, j}^{1}=1$ if $\omega^{0} \in\left[0, \beta(\cos (\theta)+\sin (\theta)]\right.$, and $\Phi_{i, j}^{1, *}=0$ otherwise.

Since $\theta \in[0, \Pi / 4]$, we have $0 \leq \beta \cos (\theta) \leq \beta\left(\cos (\theta)+\sin (\theta) \leq 1\right.$. Moreover, $\omega^{0}$ is the same for all the cells, therefore three possibilities arise:

- When $\omega^{0} \in[0, \beta \cos (\theta)]$, cells in case 1 and 2 change from 0 to 1 , and $\Phi_{i, j}^{1}$ corresponds to a translation of the initial condition of $h$ in the direction of the positive $x: \Phi_{i, j}^{1}=\Phi_{i-1, j}^{0}$.

- When $\left.\left.\omega^{0} \in\right] \beta \cos (\theta), \beta(\cos (\theta)+\sin (\theta))\right]$, only the cells in case 2 change from 0 to 1 , and $\Phi_{i, j}^{1}$ corresponds to a translation of the initial condition of $h$ in the direction of the positive $y$ : $\Phi_{i, j}^{1}=\Phi_{i, j-1}^{0}$.

- When $\left.\left.\omega^{0} \in\right] \beta(\cos (\theta)+\sin (\theta)), 1\right]$, all the cells remain unchanged, and $\Phi_{i, j}^{1}$ is equal to the initial condition: $\Phi_{i, j}^{1}=\Phi_{i, j}^{0}$.

Hence we can deduce from these remarks that, at first iteration, the initial profile is simply translated with the following transitional probabilities

$$
\left\{\begin{array}{l}
\mathcal{P}\left(\forall(i, j), \Phi_{i, j}^{1}=\Phi_{i-1, j}^{0}\right)=\beta \cos (\theta) \\
\mathcal{P}\left(\forall(i, j), \Phi_{i, j}^{1}=\Phi_{i, j-1}^{0}\right)=\beta \sin (\theta) \\
\mathcal{P}\left(\forall(i, j), \Phi_{i, j}^{1}=\Phi_{i, j}^{0}\right)=1-\beta(\cos (\theta)+\sin (\theta))
\end{array}\right.
$$

Two important points have to be quoted here in order to pursue the proof. First, the update from $t=0$ to $t=\Delta t$ preserves the initial profile in the sense that it is just translated to the right or to the top, or it remains at its initial location. The second point is that the transitional probabilities (40) only depend on the velocity field, on the time-step $\Delta t$ and on the mesh-size $h$. Since all these parameters are uniform and constant, the probabilities (40) remain unchanged for all iterations and for all cells. Hence, the iteration from a time $t^{n}$ to the time $t^{n+1}$ exactly corresponds to the first iteration in terms of transitional probabilities, and we thus obtain the probabilities (39).

For the sake of readability, let us denote by $P_{x}=\beta \cos (\theta)$ the probability to shift the approximated solution from one cell in the direction of positive $x$ and by $P_{y}=\beta \sin (\theta)$ the probability to shift the approximated solution from one cell in the direction of positive $y$.

Let us consider $N>0$ time-iterations of the Upwind-GRU scheme with (34) and (38) as an initial condition. These $N$ iterations are composed of successive $x$-shifts, $y$-shifts or standstills of the approximated initial solution. Since $P_{x}$ and $P_{y}$ do not depend on the iteration, all the combinations of the same number of shifts and standstills are equivalent. Therefore, the probability for each configuration is given by proposition 4.7.

Proposition 4.7 The probability to get exactly $K_{x}$ shifts along $x$ and $K_{y}$ shifts along $y$, with $\left(K_{x}, K_{y}\right) \in \llbracket 0, N \rrbracket^{2}$ and $K_{x}+K_{y} \leq N$, reads:

$$
P_{N}^{K_{x}, K_{y}}=C_{N}^{K_{x}, K_{y}}\left(P_{x}\right)^{K_{x}}\left(P_{y}\right)^{K_{y}}\left(1-P_{x}-P_{y}\right)^{N-K_{x}-K_{y}}
$$

where $C_{N}^{K_{x}, K_{y}}$ stands for the number of combinations:

$$
C_{N}^{K_{x}, K_{y}}=\frac{N !}{K_{x} ! K_{y} !\left(N-K_{x}-K_{y}\right) !} .
$$

Proof. As mentioned above in the proof of proposition 4.6, for each iteration we have the same transitional probabilities (39). Moreover, the random numbers $\omega^{n}$ involved in the second step of the Upwind-GRU scheme are independent for all iterations. Hence each realization is independent and follows (19). Therefore, all the configurations of $K_{x} x$-shifts, $K_{y} y$-shifts and $N-K$ standstills are equivalent in terms of probability. 
As a consequence, the configurations obtained after $N$ iterations with the Upwind-GRU scheme follow the multinomial distribution with the parameter $\left(P_{x}, P_{y}\right): \mathcal{B}\left(N, P_{x}, P_{y}\right)$. Thus, we obtain the formula (41) for the probability $P_{N}^{K_{x}, K_{y}}$ to get $K_{x} x$-shifts and $K_{y} y$-shifts for $N$ iterations.

We know from the previous proposition that the different configurations that can be obtained after $N$ iterations follow a multinomial distribution $\mathcal{B}\left(N, P_{x}, P_{y}\right)$. Since the space discretisation is known, the displacement associated with each configuration can easily be obtained. Therefore, the expectation of the displacement of the approximated initial solution after $N$ iterations, $E_{d}^{N}$, can be explicitely writen. The following result then holds.

Proposition 4.8 The expectation of the displacement of the approximated initial solution after $N$ iterations $E_{d}^{N}$ is equal to the displacement of the approximated initial solution through system (1).

Proof. Thanks to this probability (41), we can write explicitly the expectation $E_{d}^{N}=\left(E_{d, x}^{N}, E_{d, y}^{N}\right)$ of the displacement of the initial approximated solution after $N$ iterations by summing all the displacement weighted by their probabilities:

$$
E_{d}^{N}=\sum_{0 \leq K_{x}+K_{y} \leq N}\left(P_{N}^{K_{x}, K_{y}}\left(K_{x} h, K_{y} h\right)\right)
$$

The $x$-component $E_{d, x}^{N}$ of the average displacement $E_{d}^{N}$ can be written in a more convenient form:

$$
\begin{array}{r}
E_{d, x}^{N}=\sum_{0 \leq K_{x}+K_{y} \leq N}\left(P_{N}^{K_{x}, K_{y}} K_{x} h\right) ; \\
E_{d, x}^{N}=h \sum_{K_{x}=1}^{N}\left(\sum_{K_{y}=0}^{N-K_{x}}\left(K_{x} C_{N}^{K_{x}, K_{y}}\left(P_{x}\right)^{K_{x}}\left(P_{y}\right)^{K_{y}}\left(1-P_{x}-P_{y}\right)^{N-K_{x}-K_{y}}\right)\right) .
\end{array}
$$

Yet for $1 \leq K_{x} \leq N$ we have the relation:

$$
K_{x} C_{N}^{K_{x}, K_{y}}=N C_{N-1}^{K_{x}-1, K_{y}}
$$

hence the formula above for $E_{d, x}^{N}$ can be simplified in:

$$
\begin{gathered}
E_{d, x}^{N}=N h P_{x} \sum_{K_{x}=0}^{N-1}\left(\sum_{K_{y}=0}^{(N-1)-K_{x}}\left(K_{x} C_{N-1}^{K_{x}, K_{y}}\left(P_{x}\right)^{K_{x}}\left(P_{y}\right)^{K_{y}}\left(1-P_{x}-P_{y}\right)^{(N-1)-K_{x}-K_{y}}\right)\right) \\
E_{d, x}^{N}=N h P_{x} \sum_{0 \leq K_{x}+K_{y} \leq N-1}\left(C_{N-1}^{K_{x}, K_{y}}\left(P_{x}\right)^{K_{x}}\left(P_{y}\right)^{K_{y}}\left(1-P_{x}-P_{y}\right)^{(N-1)-K_{x}-K_{y}}\right) .
\end{gathered}
$$

The sum in the formula above corresponds to the development of the polynomial $\left(P_{x}+P_{y}+\left(1-P_{x}-P_{y}\right)\right)^{(N-1)}$ which is equal to 1 . So that we finally get:

$$
E_{d, x}^{N}=N h P_{x}
$$

Thanks to the definition of $P_{x}$ and $\beta$, we then obtain that:

$$
E_{d, x}^{N}=N h \beta \cos (\theta)=N \Delta t U \cos (\theta)=t^{N} U_{x}
$$

Obviously, the same computations can be done for $E_{d, y}^{N}$, the $y$-component of $E_{d}^{N}$, and it can be found that:

$$
E_{d, y}^{N}=t^{N} U_{y}
$$

As a consequence, the expectation of the displacement is equal to the exact displacement due to the velocity field $\left(U_{x}, U_{y}\right)$ :

This ends the proof of proposition (4.8).

$$
E_{d}^{N}=t^{N}\left(U_{x}, U_{y}\right)
$$

At that point, we have thus proved that each approximated solution $\Phi_{i, j}^{N}$ computed with the Upwind-GRU scheme corresponds to the initial solution $\Phi_{i, j}^{0}$ translated and that the expectation of these translations is equal to the displacement of the exact solution between time $t=0$ and time $t=t^{N}$. For the continuation of the proof, we need to study in detail the probabilities (41). In particular, it will be proved that $\left(K_{x}, K_{y}\right) \mapsto P_{N}^{K_{x}, K_{y}}$ possesses a unique maximum on $\mathcal{D}_{N}=\left\{\llbracket 0, N \rrbracket^{2}, K_{x}+K_{y} \leq N\right\}$. 
Proposition 4.9 For any number of iterations $N>N_{\beta, \theta}$, with $N_{\beta, \theta}=\max \left(1, \max \left(1 / P_{x}, 1 / P_{y}\right)-2\right)$, there exists two integers $0 \leq K_{x}^{*}<N$ and $0 \leq K_{y}^{*}<N$, such that the probability $P_{N}^{K_{x}, K_{y}}$ reaches a maximum for $\left(K_{x}^{*}, K_{y}^{*}\right)$. Moreover, the displacement associated with $\left(K_{x}^{*}, K_{y}^{*}\right)$ fulfills:

$$
\left|E_{d, x}^{N}-h K_{x}^{*}\right| \leq 2 \max \left(P_{x},\left(1-P_{x}\right)\right) h
$$

and:

$$
\left|E_{d, y}^{N}-h K_{y}^{*}\right| \leq 2 \max \left(P_{y},\left(1-P_{y}\right)\right) h
$$

Proof. For a given number of iterations $N$, the following relations between the probabilities can be obtained using simple calculus rules:

$$
\begin{aligned}
P_{N}^{K_{x}+1, K_{y}} & =P_{N}^{K_{x}, K_{y}} \frac{P_{x}\left(N-K_{x}-K_{y}\right)}{\left(1-P_{x}-P_{y}\right)\left(K_{x}+1\right)} \\
P_{N}^{K_{x}, K_{y}+1} & =P_{N}^{K_{x}, K_{y}} \frac{P_{y}\left(N-K_{x}-K_{y}\right)}{\left(1-P_{x}-P_{y}\right)\left(K_{y}+1\right)}
\end{aligned}
$$

From (48) and (49), one can obtain that:

$$
\begin{aligned}
& P_{N}^{K_{x}+1, K_{y}} \geq P_{N}^{K_{x}, K_{y}} \Longleftrightarrow N P_{x}-\left(1-P_{x}-P_{y}\right)-P_{x} K_{y}-\left(1-P_{y}\right) K_{x} \geq 0 ; \\
& P_{N}^{K_{x}, K_{y}+1} \geq P_{N}^{K_{x}, K_{y}} \Longleftrightarrow N P_{y}-\left(1-P_{x}-P_{y}\right)-\left(1-P_{x}\right) K_{y}-P_{y} K_{x} \geq 0 .
\end{aligned}
$$

Since the probabilities $P_{x}$ and $P_{y}$ do not depend on the iteration, each inequality above is afine with respect to $\left(K_{x}, K_{y}\right)$. The associated sub-domains of $\mathcal{D}_{N}$ are then separated by a line. For the first inequality, and respectively the second one, we define the lines $\left(D_{x}\right)$ and respectively $\left(D_{y}\right)$ :

$$
\begin{gathered}
\left(D_{x}\right): N P_{x}-\left(1-P_{x}-P_{y}\right)-P_{x} K_{y}-\left(1-P_{y}\right) K_{x}=0 \\
\left(D_{y}\right): N P_{y}-\left(1-P_{x}-P_{y}\right)-\left(1-P_{x}\right) K_{y}-P_{y} K_{x}=0 .
\end{gathered}
$$

These two lines are different because the determinant of the matrix that contains their normal vector reads:

$$
\left|\begin{array}{cc}
1-P_{y} & P_{x} \\
P_{y} & 1-P_{x}
\end{array}\right|=1-P_{x}-P_{y}=1-\beta(\cos (\theta)+\sin (\theta)),
$$

which is non-negative thanks to the CFL condition for $\beta$. Moreover, the two components of the normal vector of $\left(D_{x}\right)$ (resp. $\left(D_{y}\right)$ ) have the same sign which implies that $\left(D_{x}\right)$ (resp. $\left(D_{y}\right)$ ) has a negative slope in the $\left(K_{x}, K_{y}\right)$-plane. We now define the two lines that are the counterpart of $\left(D_{x}\right)$ and $\left(D_{y}\right)$ in the $(x, y)$-plane:

$$
\begin{array}{r}
\left(D_{x}^{\prime}\right): N P_{x}-\left(1-P_{x}-P_{y}\right)-P_{x} y-\left(1-P_{y}\right) x=0 ; \\
\left(D_{y}^{\prime}\right): N P_{y}-\left(1-P_{x}-P_{y}\right)-\left(1-P_{x}\right) y-P_{y} x=0 .
\end{array}
$$

The line $\left(D_{x}^{\prime}\right)$ (resp. $\left(D_{y}^{\prime}\right)$ ) has the same normal vector and the same distance to the origin $(0,0)$ than $\left(D_{x}\right)$ (resp. $\left.\left(D_{y}\right)\right)$. This distance to the origin is $N P_{x}-\left(1-P_{x}-P_{y}\right)$ for $\left(D_{x}\right)$ and $\left(D_{x}^{\prime}\right)$, and $N P_{y}-\left(1-P_{x}-P_{y}\right)$ for $\left(D_{y}\right)$ and $\left(D_{y}^{\prime}\right)$. Let us now compute $\left(x^{*}, y^{*}\right)$ the intersection point for $\left(D_{x}^{\prime}\right)$ and $\left(D_{y}^{\prime}\right)$ in the $(x, y)$-plane. It can be easily found that:

$$
x^{*}=N P_{x}-\left(1-2 P_{x}\right) \text { and } y^{*}=N P_{y}-\left(1-2 P_{y}\right) .
$$

It is an important point to be quoted that in general $x^{*}$ and $y^{*}$ do not lie in $\llbracket 0, N \rrbracket$, this explains why $\left(D_{x}^{\prime}\right)$ and $\left(D_{y}^{\prime}\right)$ have been introduced. For small $N, x^{*}$ and $y^{*}$ can be non-positive. But for any given $\beta$ and $\theta$, there exists a number of iterations $N_{\beta, \theta}=\max \left(1, \max \left(1 / P_{x}, 1 / P_{y}\right)-2\right)$ such that for all $N>N_{\beta, \theta}$ we have $x^{*} \geq 0$ and $y^{*} \geq 0$. Moreover, we have:

$$
x^{*}+y^{*} \leq N \Leftrightarrow P_{x}+P_{y} \leq 1,
$$

which is fulfilled thanks to the CFL condition for $\beta$. We assume from now that $N \geq N_{\beta, \theta}$. Therefore we get that $\left(x^{*}, y^{*}\right) \in\left\{[0, N], x^{*}+y^{*} \leq N\right\}$, the latter subset being the counterpart in $\mathbb{R}^{2}$ of the subset $\mathcal{D}_{N}$. By multiplying the coordinates $\left(x^{*}, y^{*}\right)$ by $h$, and thanks to relations (44) and (45), we obtain the associated displacement:

$$
h\left(x^{*}, y^{*}\right)=E_{d}^{N}+h\left(1-2 P_{x}, 1-2 P_{y}\right)
$$

Since $P_{x}$ and $P_{y}$ lie in $[0,1]$, we thus get:

$$
\begin{gathered}
\left|h x^{*}-E_{d, x}^{N}\right|<h \\
\left|h y^{*}-E_{d, y}^{N}\right|<h .
\end{gathered}
$$




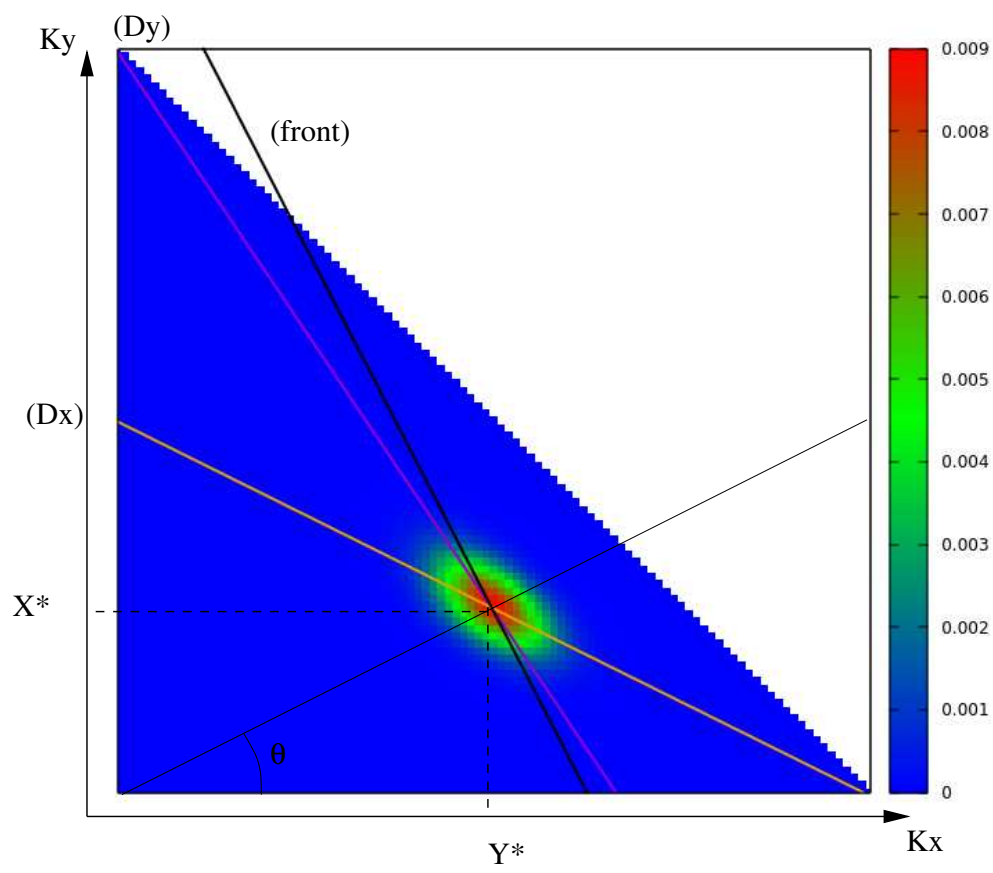

Figure 4: Probabilities $P_{N}^{K_{x}, K_{y}}$ with respect to $K_{x}$ and $K_{y}$ for $N=100, \theta=0.15 \Pi$ and $\beta=0.75 /(\cos (\theta)+$ $\sin (\theta))$. The front (black line), the lines $\left(D_{x}\right)$ (orange line) and $\left(D_{y}\right)$ (purple line), and the point $\left(x^{*}, y^{*}\right)$ are plotted.

Let us now turn back to the probabilities $P_{N}^{K_{x}, K_{y}}$. From the previous remarks, see also Fig. 4, we know that $K_{x} \mapsto P_{N}^{K_{x}, K_{y}}$ increases when $\left(K_{x}, K_{y}\right)$ is below $\left(D_{x}^{\prime}\right)$ and decreases when $\left(K_{x}, K_{y}\right)$ is above $\left(D_{x}^{\prime}\right)$; and that $K_{y} \mapsto P_{N}^{K_{x}, K_{y}}$ increases when $\left(K_{x}, K_{y}\right)$ is below $\left(D_{y}^{\prime}\right)$ and decreases when $\left(K_{x}, K_{y}\right)$ is above $\left(D_{y}^{\prime}\right)$. The intersection point of these two lines is $\left(x^{*}, y^{*}\right)$, so that the maximum of $\left(K_{x}, K_{y}\right) \mapsto P_{N}^{K_{x}, K_{y}}$ on $\mathcal{D}_{N}$ is reached at a point $\left(K_{x}^{*}, K_{y}^{*}\right)$ such that $\left|x^{*}-K_{x}^{*}\right| \leq 1$ and $\left|y^{*}-K_{y}^{*}\right| \leq 1$. Then, using relation (50), the inequality $\left|x^{*}-K_{x}^{*}\right| \leq 1$ leads to:

$$
\begin{array}{r}
-1 \leq x^{*}-K_{x}^{*} \leq 1 \\
-h \leq E_{d, x}^{N}+h\left(1-2 P_{x}\right)-h K_{x}^{*} \leq h \\
-2\left(1-P_{x}\right) h \leq E_{d, x}^{N}-h K_{x}^{*} \leq 2 P_{x} h .
\end{array}
$$

This leads to inequality (46) and, applying the same idea for the $y$-component, (47) can be found. In fact, these two inequalities state that the most probable displacement $\left(h K_{x}^{*}, h K_{y}^{*}\right)$ following $P_{N}^{K_{x}, K_{y}}$ tends towards the exact displacement $E_{d}^{N}$ when the mesh size $h$ tends towards zero (at a fixed $\beta$ ). This ends the proof of proposition 4.9 .

Let us choose $\eta>0$, and let us define the subset of $\mathcal{D}_{N}$ :

$$
\mathcal{H}_{N}(\eta)=\left\{\left(K_{x}, K_{y}\right) \in \mathcal{D}_{N} /\left|E_{d, x}^{N}-h K_{x}\right| \leq \eta \quad \text { and } \quad\left|E_{d, y}^{N}-h K_{y}\right| \leq \eta\right\}
$$

The subset $\mathcal{H}_{N}(\eta)$ contains all the possible couples $\left(K_{x}, K_{y}\right)$ that, for $N$ iterations, lead to a displacement which have a distance from the exact displacement less than $\eta$. Since we have a cartesian setting, the distance used here corresponds to a $L^{1}$-norm component by component. Let us fix a final time $T$ and let us fix a CFL number $\beta$ in $] 0,(\cos (\theta)+\sin (\theta))^{-1}[$. For a given number of iterations $N$, the time-step is chosen constant: $\Delta t=T / N$. We thus get that the mesh size if fixed and reads: $h=T U /(N \beta)$.

Proposition 4.10 Let us assume that the final time $T$ and the $C F L$ number $\beta \in] 0,(\cos (\theta)+\sin (\theta))^{-1}[$ are given. Let $N$ be the total number of time-steps for reaching the final time $T$, so that both $h$ and $\Delta t$ are known for any number of iterations $N$. The displacement of the approximated initial solution by the Upwind-GRU scheme after $N$ time-iterations converges almost surely to the mean displacement $E_{d}^{N}=\left(E_{d, x}^{N}, E_{d, y}^{N}\right)=\left(T U_{x}, T U_{y}\right)$ as $N$ tends towards $+\infty$ :

$$
\left(\mathcal{K}_{x}^{N} h, \mathcal{K}_{y}^{N} h\right) \stackrel{a s}{\longrightarrow}\left(T U_{x}, T U_{y}\right),
$$

where $\left(\mathcal{K}_{x}^{N}\right)$ (resp. $\left.\left(\mathcal{K}_{y}^{N}\right)\right)$ denotes the random variable associated with the number of shifts of the approximated solution in the $x$-direction (resp. $y$-direction). 
Proof. We first define $\mathcal{P}_{N}^{\eta}$, the probability for the displacement of the approximated initial solution to be in the domain $\left[E_{d, x}^{N}-\eta, E_{d, x}^{N}+\eta\right] \times\left[E_{d, y}^{N}-\eta, E_{d, y}^{N}+\eta\right]$ after $N$ time-iterations. Inequalities (46) and (47) imply that, for a given $h$, there exists $N_{\eta}>N_{\beta, \theta}$ such that for all $N>N_{\eta}$ we have $\left(K_{x}^{*}, K_{y}^{*}\right) \in \mathcal{H}_{N}(\eta)$. In the following, we assume that we have $N>N_{\eta}$.

From the previous results (see inequalities (51)-(52) and the results of monotony for $P_{N}^{K_{x}, K_{y}}$ on $\mathcal{D}_{N}$ ) we can deduce that there exists a couple $\left(K_{x, m}, K_{y, m}\right)$ in $\mathcal{D}_{N} \backslash \mathcal{H}_{N}(\eta)$ such that:

$$
\forall\left(K_{x}, K_{y}\right) \in \mathcal{D}_{N} \backslash \mathcal{H}_{N}(\eta), P_{N}^{K_{x}, K_{y}} \leq P_{N}^{K_{x, m}, K_{y, m}}
$$

Due to the monotony results, this maximum probability $P_{N}^{K_{x, m}, K_{y, m}}$ is always reached close to the frontier between $\mathcal{H}_{N}(\eta)$ and $\mathcal{D}_{N} \backslash \mathcal{H}_{N}(\eta)$, which means that we have:

$$
\eta<\left|E_{d, x}^{N}-h K_{x, m}\right| \leq \eta+h, \quad \text { and } \quad \eta<\left|E_{d, y}^{N}-h K_{y, m}\right| \leq \eta+h
$$

As a consequence, by using (44) and the relation $h=T U /(N \beta)$, the first inequality of (53) gives:

$$
\begin{array}{r}
E_{d, x}^{N}-\eta-h \leq h K_{x, m} \leq E_{d, x}^{N}+\eta+h \\
h N P_{x}-\eta-h \leq h K_{x, m} \\
N \beta\left(\cos (\theta)-\frac{\eta}{T U}\right) \leq K_{x, m}+1
\end{array}
$$

In the same way, one can easily find from the second inequality of (53) that:

$$
N \beta\left(\sin (\theta)-\frac{\eta}{T U}\right) \leq K_{y, m}+1
$$

We can deduce from inequalities (54) and (55), and from the fact that the parameters $\theta, T$ and $U$ are fixed, that for small enough values of $\eta$ the integers $K_{x, m}$ and $K_{y, m}$ tend towards $+\infty$ linearly when $N$ tends towards $+\infty$. According to (54) and (55), this is the case if the following sufficient condition is fulfilled: $\eta \leq \min \left(U_{x}, U_{y}\right) T$. From now we thus assume that $\eta$ fulfills that condition.

The probability to have a couple $\left(\mathcal{K}_{x}^{N}, \mathcal{K}_{y}^{N}\right)$ in $\mathcal{D}_{N} \backslash \mathcal{H}_{N}(\eta)$ is thus:

$$
\mathcal{P}\left(\left(\mathcal{K}_{x}^{N}, \mathcal{K}_{y}^{N}\right) \in \mathcal{D}_{N} \backslash \mathcal{H}_{N}(\eta)\right)=\sum_{\left(K_{x}, K_{y}\right) \in \mathcal{D}_{N} \backslash \mathcal{H}_{N}(\eta)} P_{N}^{K_{x}, K_{y}}<\operatorname{card}\left(\mathcal{D}_{N} \backslash \mathcal{H}_{N}(\eta)\right) P_{N}^{K_{x, m}, K_{y, m}}
$$

Since $\operatorname{card}\left(\mathcal{D}_{N} \backslash \mathcal{H}_{N}(\eta)\right) \leq N^{2}$, we get the inequality:

$$
\mathcal{P}\left(\left(\mathcal{K}_{x}^{N}, \mathcal{K}_{y}^{N}\right) \in \mathcal{D}_{N} \backslash \mathcal{H}_{N}(\eta)\right)<N^{2} P_{N}^{K_{x, m}, K_{y, m}},
$$

and by expressing explicitly $P_{N}^{K_{x, m}, K_{y, m}}$ we obtain:

$$
\mathcal{P}\left(\left(\mathcal{K}_{x}^{N}, \mathcal{K}_{y}^{N}\right) \in \mathcal{D}_{N} \backslash \mathcal{H}_{N}(h)\right)<N^{2} C_{N}^{K_{x, m}, K_{y, m}}\left(P_{x}\right)^{K_{x, m}}\left(P_{y}\right)^{K_{y, m}}\left(1-P_{x}-P_{y}\right)^{N-K_{x, m}-K_{y, m}} .
$$

When $N$ tends towards $+\infty$, the term $N^{2} C_{N}^{K_{x, m}, K_{y, m}}$ on the right hand side of the inequality tends to $+\infty$ as a polynomial in $N$; whereas the three other terms tend to zero as exponential terms (since $P_{x}$ and $P_{y}$ are in $[0,1])$. Hence, we finally get that

$$
\mathcal{P}\left(\left(\mathcal{K}_{x}^{N}, \mathcal{K}_{y}^{N}\right) \in \mathcal{D}_{N} \backslash \mathcal{H}_{N}(\eta)\right) \underset{N \rightarrow+\infty}{\longrightarrow} 0
$$

and obviously we have

$$
\mathcal{P}\left(\left(\mathcal{K}_{x}^{N}, \mathcal{K}_{y}^{N}\right) \in \mathcal{H}_{N}(\eta)\right) \underset{N \rightarrow+\infty}{\longrightarrow} 1
$$

The latter means that for any given $\eta \leq \min \left(U_{x}, U_{y}\right) T$, the probability to have a displacement inside $\mathcal{H}_{N}(\eta)$ tends to 1. Due to our choice, $E_{d}^{N}$ does not depend on $N: E_{d}^{N}=t^{N}\left(U_{x}, U_{y}\right)=T\left(U_{x}, U_{y}\right)$. Thus the frontier of the domain $\mathcal{H}_{N}(\eta)$ which is given by $h$ and $E_{d}^{N}$, does not depend on $N$. We can then deduce from (58) that:

$$
\left.\mathcal{P}_{N}^{\eta}=\mathcal{P}\left(\left|\mathcal{K}_{x}^{N} h-T U_{x}\right|<\eta \text { and }\left|\mathcal{K}_{y}^{N} h-T U_{y}\right|<\eta\right)\right)=\mathcal{P}\left(\left(\mathcal{K}_{x}^{N}, \mathcal{K}_{y}^{N}\right) \in \mathcal{H}_{N}(\eta)\right) \underset{N \rightarrow+\infty}{\longrightarrow} 1
$$

Since the limit (59) holds for all $0<\eta \leq \min \left(U_{x}, U_{y}\right) T$, we can conclude that the approximated displacement associated with the Upwind-GRU scheme tends towards the exact displacement with probability 1 when the number of iterations tends towards $+\infty$ (i.e. when the time-step $\Delta t$ or the mesh-size $h$ tend towards 0 ). 
Moreover, if we sum the inequality (56) for $N=1$ to $N=N_{0} \geq 1$, we get that:

$$
\sum_{N=1}^{N_{0}} \mathcal{P}\left(\left(\mathcal{K}_{x}^{N}, \mathcal{K}_{y}^{N}\right) \in \mathcal{D}_{N} \backslash \mathcal{H}_{N}(h)\right)<\sum_{N=1}^{N_{0}} N^{2} C_{N}^{K_{x, m}, K_{y, m}}\left(P_{x}\right)^{K_{x, m}}\left(P_{y}\right)^{K_{y, m}}\left(1-P_{x}-P_{y}\right)^{N-K_{x, m}-K_{y, m}} .
$$

Since the term $N^{2} C_{N}^{K_{x, m}, K_{y, m}}$ tends to $+\infty$ as a polynomial in $N$, and since the three other terms tend to zero as exponential terms (since $P_{x}$ and $P_{y}$ are in $[0,1]$ ), the sum on the left-hand-side of inequality (60) remains bounded when $N_{0}$ tends towards $+\infty$. Therefore, there exists a constant $C_{2}>0$ such that:

$$
\sum_{N=1}^{+\infty} \mathcal{P}\left(\left(\mathcal{K}_{x}^{N}, \mathcal{K}_{y}^{N}\right) \in \mathcal{D}_{N} \backslash \mathcal{H}_{N}(h)\right)<C_{2}
$$

whereof we deduce thanks to the Borel-Cantelli's theorem that the random variable $\left(\mathcal{K}_{x}^{N} h, \mathcal{K}_{y}^{N} h\right)$ converges almost surely to $\left(E_{d, x}^{N}, E_{d, y}^{N}\right)=\left(T U_{x}, T U_{y}\right)$ when $N$ tends towards $+\infty$ :

$$
\left(\mathcal{K}_{x}^{N} h, \mathcal{K}_{y}^{N} h\right) \stackrel{a s}{\longrightarrow}\left(T U_{x}, T U_{y}\right) .
$$

This ends the proof of proposition 4.10 .

In the following, we assume that the final time $T$ and the CFL number $\beta \in] 0,(\cos (\theta)+\sin (\theta))^{-1}[$ are given, so that both $h$ and $\Delta t$ are known for any number of iterations $N$. Let us define the front of the approximated solution. Obviously, the latter depends on the number of iterations $N$. It reads:

$$
\mathcal{F}^{N, g r u}=\mathcal{F}_{x}^{N, g r u} \cup \mathcal{F}_{y}^{N, g r u},
$$

where the set $\mathcal{F}_{x}^{N, g r u}$ (resp. $\mathcal{F}_{y}^{N, g r u}$ ) corresponds to all the faces along $x$ (resp. along $y$ ) which separate two cells associated with different values of the approximated solution a iteration $N$ :

$$
\mathcal{F}_{x}^{N, g r u}=\bigcup_{(i, j)}\left\{\left[x_{i-1 / 2}, x_{i+1 / 2}\right] \times\left\{y_{j-1 / 2}\right\}, \Phi_{i, j-1}^{N} \neq \Phi_{i, j}^{N}\right\}
$$

and

$$
\mathcal{F}_{y}^{N, g r u}=\bigcup_{(i, j)}\left\{\left\{x_{i-1 / 2}\right\} \times\left[y_{j-1 / 2}, y_{j+1 / 2}\right], \Phi_{i-1, j}^{N} \neq \Phi_{i, j}^{N}\right\} .
$$

Finally, $\mathcal{F}^{N, g r u}$ contains all the points of $\mathbb{R}^{2}$ that belong to the front of the approximated solution. Moreover, thanks to the definition of the approximated initial solution (38) and to the choices for $U$ and $n$, all the cells $(i, j)$ that are below $\mathcal{F}^{0, g r u}$ with respect to the $y$-axis (or equivalently that are on the left of $\mathcal{F}^{0, g r u}$ with respect to the $x$-axis) are such that $\Phi_{i, j}^{N}=1$. Conversely, all the other cells are such that $\Phi_{i, j}^{N}=0$. Thanks to the definition of the exact solution (35) we can easily define the front $\mathcal{F}(t)$ of the exact solution at time $t$ as the translation due to the velocity $U=\left(U_{x}, U_{y}\right)$ of the initial front:

$$
D_{f}^{0}=\left\{(x, y) ;\left(x-X_{f}^{0}\right) \cos (\theta)+\left(y-Y_{f}^{0}\right) \sin (\theta)=0\right\},
$$

which has already been defined at the beginning of Section 4.3. We thus have:

$$
\mathcal{F}(t)=D_{f}^{0}+t\left(U_{x}, U_{y}\right) .
$$

We also define the following distance between two connected subsets $A$ and $B$ of $\mathbb{R}^{2}$ :

$$
d(A, B)=\sup _{x \in A}\left(\inf _{y \in B}(|x-y|)\right)+\sup _{y \in B}\left(\inf _{x \in A}(|x-y|)\right) .
$$

It should be noted that, if $A \subset B$ (resp. $B \subset A$ ) then the second term of $d(A, B)$ (resp. the first term of $d(A, B))$ vanishes. Hence, we have: $A=B \Longrightarrow d(A, B)=0$. Moreover, if $d(A, B)=0$ the two terms in the definition of the distance are equal to zero, which implies that:

$$
\forall x \in A, \underset{y \in B}{\inf }(|x-y|)=0, \quad \text { and } \quad \forall y \in B, \inf _{x \in A}(|x-y|)=0,
$$

and, thus:

which leads to:

$$
\forall x \in A, \forall y \in B, x=y ; \quad \text { and } \forall y \in B, \forall x \in A, x=y
$$

$\forall x \in A, x \in B, \quad$ and $\quad \forall y \in B, y \in A$.

Therefore, we have: $d(A, B)=0 \Longrightarrow A=B$. So that we can conclude that $d(A, B)=0 \Longleftrightarrow A=B$.

Since the result of proposition 4.10 remains true for any $\eta>0$, it can be stated that the displacement due to the Upwind-GRU scheme tends towards the exact displacement with probability 1 when the number of iterations $N$ tends towards $+\infty$. We can then conclude the whole proof with the following theorem. 
Theorem 4.2 Let us assume that the final time $T$ and the $C F L$ number $\beta \in] 0,(\cos (\theta)+\sin (\theta))^{-1}[$ are given. Let $N$ be the total number of time-steps for reaching the final time $T$, so that both $h$ and $\Delta t$ are known for any number of iterations $N$. The approximated solution obtained by the Upwind-GRU scheme after $N$ timeiterations converges almost surely to the exact solution at time $T$ when $N$ tends towards $+\infty$ when considering the distance $d(.,$.$) in the sense that:$

$$
d\left(\mathcal{F}^{N, g r u}, \mathcal{F}(T)\right) \stackrel{a s}{\longrightarrow} 0 .
$$

Proof. For a final time $T$ and a CFL number $\beta \in] 0,(\cos (\theta)+\sin (\theta))^{-1}[$, both $h$ and $\Delta t$ are known for any number of iterations $N$ through the relations: $\Delta t=T / N$ and $h=T U /(N \beta)$. At time $T$, the exact solution (35) corresponds to the translation with the uniform vector $U \times T$ of the initial condition (34), so that we have for the exact front at time $T$ :

$$
\mathcal{F}(T)=\mathcal{F}(0)+U \times T
$$

It is an important point to be recalled here that the same random number $\omega^{k}$ is used for all the cells at iteration $k$. Therefore, one time-step of the Upwind-GRU scheme corresponds to a translation $(+h, 0)$ or $(0,+h)$ of the approximated solution, see proposition 4.6. We denote by $V^{N, g r u}$ the displacement due to the UpwindGRU scheme after $N$ iterations, which is composed of $\mathcal{K}_{x}^{N}$ displacement along $x$ and $\mathcal{K}_{y}^{N}$ displacement along $y$ : $V^{N, g r u}=\left(\mathcal{K}_{x}^{N} h, \mathcal{K}_{y}^{N} h\right)$. We know from proposition 4.8, that it is uniform and that its expectation is equal to $U \times T=\left(U_{x} T, U_{y} T\right)$. We then have:

$$
\mathcal{F}^{N, g r u}=\mathcal{F}^{0, g r u}+V^{N, g r u} .
$$

Considering equations (64) and (65) we get:

$$
d\left(\mathcal{F}(T), \mathcal{F}^{N, g r u}\right)=d\left(\mathcal{F}(0)+U T, \mathcal{F}^{0, g r u}+V^{N, g r u}\right) .
$$

Since the translation vectors $U T$ and $V^{N, g r u}$ are uniform, the definition of the distance (63) leads to:

$$
d\left(\mathcal{F}(T), \mathcal{F}^{N, g r u}\right)=d\left(\mathcal{F}(0), \mathcal{F}^{0, g r u}\right)+\left|U T-V^{N, g r u}\right| .
$$

Thanks to our choice for the initial condition, the equality above gives:

$$
d\left(\mathcal{F}(T), \mathcal{F}^{N, g r u}\right) \leq h+\left|U \times T-V^{N, g r u}\right|=\frac{T U}{\beta N}+\left|U T-V^{N, g r u}\right|,
$$

Since $T, U$ and $\beta$ are fixed, when $N$ tends towards $+\infty$, we have $T U /(\beta N) \rightarrow 0$. Moreover, thanks to proposition 4.10 we also have $\left|U T-V^{N, g r u}\right| \stackrel{a s}{\longrightarrow} 0$. It can therefore be concluded that:

$$
d\left(\mathcal{F}(T), \mathcal{F}^{N, g r u}\right) \stackrel{a s}{\longrightarrow} 0
$$

when $N$ tends towards $+\infty$. This proves that the approximated front converges almost surely towards the exact front when $N$ tends towards $+\infty$ when considering the distance $d(.,$.$) . This ends the proof of theorem 4.2.$

Finally, we have proved that the approximated solutions computed with the Upwind-GRU scheme converge almost surely towards the exact solution for the specific problem involving: a constant and uniform velocity and an initial condition composed of two states separated by a linear front. Obviously, it is possible to extend this result to the three-dimensional case when focusing of the same kind of configurations. For more complex configurations the same limitations are encountered as those shortly described at the end of Section 4.2 concerning the one-dimensional case.

\subsection{Three-dimensional case}

The results of Section (4.3) can be straightforwardly extended to the three-dimensional case. The same arguments can be used even if the calculus may be a little more complex. We thus do not develop entirely here the three-dimensional case, and we limit this section to the statement of the counterpart of theorem (4.2) for the convergence of the Upwind-GRU scheme. We consider here a uniform mesh composed of cubic cells with a length $h$. The set of equations is now:

$$
\left\{\begin{array}{l}
\partial_{t} \Phi(t, x, y, z)+U_{x} \partial_{x} \Phi(t, x, y, z)+U_{y} \partial_{y} \Phi(t, x, y, z)+U_{z} \partial_{z} \Phi(t, x, y, z)=0 \\
\Phi(t=0, x, y, z)=\Phi^{0}(x, y, z)
\end{array}\right.
$$

where the velocity field $\left(U_{x}, U_{y}, U_{Z}\right)$ is such that $U_{x}>0, U_{y}>0$ and $U_{z}>0$. As in the previous section, we choose a specific initial condition associated with a planar front:

$$
\Phi^{0}(x, y, z)= \begin{cases}1 & \text { if }\left(x-X_{f}^{0}\right) n_{x}+\left(y-Y_{f}^{0}\right) n_{y}+\left(z-Z_{f}^{0}\right) n_{z}<0 \\ 0 & \text { otherwise. }\end{cases}
$$


where $n=\left(n_{x}, n_{y}, n_{z}\right)$ is the normal vector to the initial front and $\left(X_{f}^{0}, Y_{f}^{0}, Z_{f}^{0}\right)$ a reference point of the initial front. As in Section (4.3), we assume that $n_{x}^{2}+n_{y}^{2}+n_{z}^{2}=1$ with $n_{x}>0, n_{y}>0$ and $n_{z}>0$. The approximated initial condition $\Phi_{i, j, k}^{0}$ for the Upwind-GRU scheme is defined as:

$$
\Phi_{i, j, k}^{0}=\Phi^{0}\left(X_{i}, Y_{j}, Z_{k}\right),
$$

where $\left(X_{i}, Y_{j}, Z_{k}\right)$ corresponds to the center of gravity of cell $(i, j, k)$. As for the two-dimensional case, see definition (63), we define the following distance between two connected subsets $A$ and $B$ of $\mathbb{R}^{3}$ :

$$
d(A, B)=\sup _{x \in A}\left(\inf _{y \in B}(|x-y|)\right)+\sup _{y \in B}\left(\inf _{x \in A}(|x-y|)\right) .
$$

For the problem defined in this section, theorem 4.3 then states that the approximated solution obtained with the Upwind-GRU scheme converge towards the exact solution.

Theorem 4.3 Let us assume that the final time $T$ and the CFL number $\beta \in] 0,\left(n_{x}+n_{y}+n_{z}\right)^{-1}[$ are given. Let $N$ be the total number of time-steps for reaching the final time $T$, so that both $h$ and $\Delta t$ are known for any number of iterations $N$. The approximated solution obtained by the Upwind-GRU scheme after $N$ timeiterations converges almost surely to the exact solution at time $T$ when $N$ tends towards $+\infty$ when considering the distance $d(.,$.$) in the sense that:$

$$
d\left(\mathcal{F}^{N, g r u}, \mathcal{F}(T)\right) \stackrel{a s}{\longrightarrow} 0 .
$$

\subsection{A shorter proof for the convergence in probability}

For the sake of simplicity, the following proof is proposed for the two-dimensional case but is obviously can be extended to the one- and three-dimensional cases. The present proof is much shorter that the one proposed in Section 4.3. Indeed it relies on some classical and general results of probability, as the weak law of large numbers, instead of using exact computations. As in Section 4.3, for a final time $T$ and a CFL number $\beta \in] 0,(\cos (\theta)+\sin (\theta))^{-1}[$, both $h$ and $\Delta t$ are known for any number of iterations $N$ through the relations: $\Delta t=T / N$ and $h=T U /(N \beta)$.

We consider here the assumptions of Section 4.3. Let us first recall that the approximated front $\mathcal{F}^{N, g r u}$ at iteration $N$ has been defined by (61). Using definition (70), we get that the distance $d(.,$.$) between the$ approximated initial front and the exact front fulfills the relation:

$$
d\left(\mathcal{F}^{0, g r u}, D_{f}^{0}\right) \leq h,
$$

which is obviously consistent with relation (37). We then have the following result.

Theorem 4.4 Let us assume that the final time $T$ and the $C F L$ number $\beta \in] 0,(\cos (\theta)+\sin (\theta))^{-1}[$ are given, so that both $h$ and $\Delta t$ are known for any number of iterations $N$. The approximated front computed with the Upwind-GRU scheme converges in probability towards the exact front with order 1 with respect to $h$ when $h \rightarrow 0$.

Proof. At time $t=T$, the exact front $D_{f}(t)$ associated with the exact solution (35) is equal to the initial front translated by $\left(U_{x} T, U_{y} T\right)$ :

$$
D_{f}(t)=D_{f}^{0}+T\left(U_{x}, U_{y}\right) .
$$

From the previous sections, we know that $\mathcal{F}^{N, g r u}$ is obtained by successive translations of vector: $(0,0),(h, 0)$ or $(0, h)$. For each iteration $n$, the choice between these different possible translations is governed by the random number $\omega^{n}$. We define $\delta D_{n}$ the random variable corresponding to the translation at an iteration $n$ in the direction of the velocity field $\left(U_{x}, U_{y}\right)$. We know that:

$$
\left\{\begin{array}{l}
\mathcal{P}\left(\delta D_{n}=h \cos (\theta)\right)=\beta \cos (\theta)=P_{x} \\
\mathcal{P}\left(\delta D_{n}=h \sin (\theta)\right)=\beta \sin (\theta)=P_{y}, \\
\mathcal{P}\left(\delta D_{n}=0\right)=1-P_{x}-P_{y} .
\end{array}\right.
$$

Hence, the variables $\left(\delta D_{n}\right)$ are independent and they all follow the same generalized Bernoulli law with parameters $P_{x}$ and $P_{y}$. As a classical result, we get that the expectation and the variance of the variable $\delta D^{n}$ are respectively:

$$
E\left[\delta D^{n}\right]=h \beta, \quad \text { and } \quad \operatorname{Var}\left(\delta D^{n}\right)=h^{2} \beta v(\beta, \theta),
$$

with $v(\beta, \theta)=\left(\cos (\theta)^{3}+\sin (\theta)^{3}-\beta\right) \geq 0$ thanks to the choices $\beta<(\cos (\theta)+\sin (\theta))^{-1}$ and $\left.\theta \in\right] 0, \Pi / 4[$. 
As a consequence, the displacement in the direction of the velocity $\left(U_{x}, U_{y}\right)$ of the initial approximated front after $N$ iterations, $X^{N}=\sum_{i=1}^{N} \delta D_{i}$, is a random variable that follows a multinomial law of parameter $\left(P_{x}, P_{y}\right)$. We thus obtain the probability for $X^{N}$ :

$$
P\left(X^{N}=K_{x} h \cos (\theta)+K_{y} h \sin (\theta)\right)=C_{N}^{K_{x}, K_{y}}\left(P_{x}\right)^{K_{x}}\left(P_{y}\right)^{K_{y}}\left(1-P_{x}-P_{y}\right)^{N-K_{x}-K_{y}},
$$

with $\left(K_{x}, K_{y}\right)$ a couple of positive integers such that $K_{x}+K_{Y} \leq N$. As in Section $4.3, K_{x}$ (respectively $K_{y}$ ) corresponds to the number of translations $(h, 0)$ (resp. $(0, h)$ ). Equation $(74)$ is the counterpart of equation (41) given in proposition 4.7. The analogous of the result of proposition 4.8 is then:

$$
E\left[X^{N}\right]=T U
$$

If we define $\bar{X}^{N}=X^{N} / N$, which corresponds to the average displacement per iteration in the direction of the velocity $\left(U_{x}, U_{y}\right)$ for $N$ iterations, we can apply the weak law of large numbers to the variables $\left(\delta D^{n}\right)$. The latter states that, since $\left(\delta D^{n}\right)$ are independent and identically distributed variables, $\bar{X}^{N}$ is such that:

$$
E\left[\bar{X}^{N}\right]=E\left[\delta D^{n}\right]=\beta h
$$

which is in equivalent to $(75)$ when $N \beta h=T U$, and:

$$
\operatorname{Var}\left(\bar{X}^{N}\right)=\frac{\operatorname{Var}\left(\delta D^{n}\right)}{N}=\frac{h^{2} \beta}{N} v(\beta, \theta)
$$

where $\operatorname{Var}($.$) denotes the variance. Moreover, the Chebyshev inequality provides the relation:$

$$
\forall \epsilon>0, \quad P\left(\left|\bar{X}^{N}-E\left[\bar{X}^{N}\right]\right| \geq \epsilon\right) \leq \frac{\operatorname{Var}\left(\bar{X}^{N}\right)}{\epsilon^{2}},
$$

whereof we deduce, by setting $\epsilon^{\prime}=N \epsilon$ and by using (76) and (77), that:

$$
\forall \epsilon^{\prime}>0, \quad P\left(\left|X^{N}-N \beta h\right| \geq \epsilon^{\prime}\right) \leq \frac{N h^{2} \beta}{\left(\epsilon^{\prime}\right)^{2}} .
$$

Hence, since $1 / N=\beta h /(T U)$ relation (78) leads to:

$$
\forall \epsilon^{\prime}>0, \quad P\left(\left|X^{N}-T U\right| \geq \epsilon^{\prime}\right) \leq \frac{(T U)^{2}}{\left(\epsilon^{\prime}\right)^{2} N}=\frac{(T U) \beta}{\left(\epsilon^{\prime}\right)^{2}} h .
$$

The quantities $\beta$ and $T U$ are fixed, so we get from (79) that the approximated displacement in the direction of the velocity $\left(U_{x}, U_{y}\right)$ obtained with the Upwind-GRU scheme converges in probability towards the exact one when the number of iterations tends to $+\infty: X^{N} \stackrel{p}{\rightarrow} T U$. Moreover, thanks to the relation between $N$ and $h$, we get that $X^{N}$ converges in probability to $T U$ with order 1 with respect to $h$ when $h \rightarrow 0$.

We can then conclude following the same idea than that used in the proof of theorem 4.2. The approximated front $\mathcal{F}^{N, g r u}$ at iteration $N$ is the set:

$$
\mathcal{F}^{N, g r u}=\mathcal{F}^{0, g r u}+X^{N}(\cos (\theta), \sin (\theta)) .
$$

Yet, for a final time $T$ and a CFL number $\beta \in] 0,(\cos (\theta)+\sin (\theta))^{-1}[$, both $h$ and $\Delta t$ are known for any number of iterations $N$ through the relations: $\Delta t=T / N$ and $h=T U /(N \beta)$. Thanks to (71), when $h$ tends towards $0, \mathcal{F}^{0, g r u}$ tends to $D_{f}^{0}$ surely with order 1 in $h$ (this convergence is deterministic). Moreover, according to (79), $X^{N}(\cos (\theta), \sin (\theta))$ tends with probability 1 to :

$$
T U(\cos (\theta), \sin (\theta))=T\left(U_{x}, U_{y}\right)
$$

with order 1 with respect to $h$. By considering the distance $d(.,$.$) , we can then conclude that the approximated$ front converges in probability with order $1 \mathrm{in} h$ towards the exact front (72) when $h$ tends towards 0 . This ends the proof of theorem 4.4 .

It should be noted that inequality (79) is less sharp (at least for large enough $N$ ) that the inequality obtained in Section 4.3. In particular (79) is not sufficient to allow the use of the Borel-Cantelli's theorem for obtaining the convergence almost surely. Obviously, (74) provides a sharper bound as in Section 4.3. This allows to apply for the Borel-Cantelli's theorem and to get the convergence almost surely. 


\section{Conclusion}

Some results and properties of the Upwind-GRU scheme introduced in [8] have been studied. In particular, a proof of convergence of the Upwind-GRU scheme has been proposed considering a two-dimensional and a three-dimensional setting and a particular class of advection problems. The latter corresponds to the transport of a planar front by a constant and uniform velocity field.

It is an important point to be quoted here that the choice of the same random number $\omega^{k}$ for all the cells at iteration $k$ appears as the key point in order to get a consistent scheme. This is clearly highlighted by the different results proposed in this work. Moreover, the proofs of Section 4 are based on the properties of the multinomial probability distribution which arises from the assumption that the velocity field is constant and uniform. If this assumption does not hold, the proof may become more complex. Indeed, expressing a monotony result as the one stated in proposition 4.9 becomes very tricky. This would be the case when studying extensions to non-uniform Cartesian grids and/or non-uniform velocity fields. For such cases, the use of more general results could be of great help, as briefly shown in Section 4.5. The weak law of large numbers has been used in Section 4.5 thanks to our specific situation, but the strong law of large numbers or the central limit theorem might be efficient mathematical tools in order to treat the more complex situations, as in [4] or [10].

Nevertheless, some extensions of the proof of Sections 4.2, 4.3 and 4.4 to more general cases could be possible, at least for uniform Cartesian grids. For instance, initial solutions involving a non-planar front could also be considered provided that the components of its local normal vector have the same sign everywhere. For such cases, the same arguments than that developed in Section 4 could be used. Nonetheless when the sign of at least one of the components of the local normal vector changes, the approximated solutions may undergo the pathological behavior reported in Section 3.2. Therefore, this should be accounted for in the proof.

At last, it should be mentioned that more complex situations have been investigated numerically in [8]. Test cases involving non-uniform meshes and non-uniform velocity fields have been considered. For each of these complex configurations, the approximated solutions computed using the Upwing-GRU scheme converge towards the exact solution with an effective convergence rate of 0.8 when the mesh is refined.

\section{Acknowledgments}

The authors want to thank Raphaèle Herbin and Jean-Marc Hérard for the sessions of fruitful discussions.

\section{References}

[1] A. J. Chorin, Random choice solution of hyperbolic systems, Journal of Computational Physics, 22 (1976), pp. $517-533$.

[2] P. Colella, Analysis of the effect of operator splitting and of the sampling procedure on the accuracy of glimm's method, Report No. LBL-8774. California Univ., Berkeley (USA). Lawrence Berkeley Lab., (1978).

[3] G Glimm's method for gas dynamics, SIAM Journal on Scientific and Statistical Computing, 3 (1982), pp. $76-110$.

[4] F. Delarue and F. Lagoutière, Probabilistic analysis of the upwind scheme for transport equations, Archive for rational mechanics and analysis, 199 (2011), pp. 229-268.

[5] J. Guimm, Solutions in the large for nonlinear hyperbolic systems of equations, Communications on pure and applied mathematics, 18 (1965), pp. 697-715.

[6] A. Harten, P. D. Lax, And B. V. Leer, On upstream differencing and Godunov-type schemes for hyperbolic conservation laws, SIAM review, 25 (1983), pp. 35-61.

[7] P. Helluy and J. Jung, Interpolated pressure laws in two-fluid simulations and hyperbolicity, Finite Volumes for Complex Applications VII-Methods and Theoretical Aspects, (2014), pp. 37-53.

[8] O. Hurisse, On the use of Glimm-like schemes for transport equations on multi-dimensional domain, Int. Journal for Numerical Methods in Fluids, (2020), p. available online.

[9] T.-P. Liu, The deterministic version of the Glimm scheme, Commun. math. Phys., 57 (1977), pp. 135-148.

[10] A. Schlichting And C. SeIs, Convergence rates for upwind schemes with rough coefficients, SIAM J. Numer. Anal., 55 (2017), pp. 812-840.

[11] D. SERRE, Systems of Conservation Laws 1: Hyperbolicity, entropies, shock waves, Cambridge University Press, 1999. 